\title{
Efficient Brownian Dynamics of rigid colloids in linear flow fields based on the grand mobility matrix
}

\author{
Duraivelan Palanisamy and Wouter K. den Otter ${ }^{\mathrm{a})}$ \\ Multi-Scale Mechanics, Faculty of Engineering Technology and MESA+ Institute for Nanotechnology, \\ University of Twente, P.O. Box 217, 7500 AE Enschede, The Netherlands
}

(Received 27 February 2018; accepted 2 May 2018; published online 21 May 2018)

\begin{abstract}
We present an efficient general method to simulate in the Stokesian limit the coupled translational and rotational dynamics of arbitrarily shaped colloids subject to external potential forces and torques, linear flow fields, and Brownian motion. The colloid's surface is represented by a collection of spherical primary particles. The hydrodynamic interactions between these particles, here approximated at the Rotne-Prager-Yamakawa level, are evaluated only once to generate the body's $(11 \times 11)$ grand mobility matrix. The constancy of this matrix in the body frame, combined with the convenient properties of quaternions in rotational Brownian Dynamics, enables an efficient simulation of the body's motion. Simulations in quiescent fluids yield correct translational and rotational diffusion behaviour and sample Boltzmann's equilibrium distribution. Simulations of ellipsoids and spherical caps under shear, in the absence of thermal fluctuations, yield periodic orbits in excellent agreement with the theories by Jeffery and Dorrepaal. The time-varying stress tensors provide the Einstein coefficient and viscosity of dilute suspensions of these bodies. Published by AIP Publishing. https://doi.org/10.1063/1.5027063
\end{abstract}

\section{INTRODUCTION}

Colloidal suspensions are ubiquitous in nature and in man-made materials. The dynamics of colloidal particles are therefore of interest to both academia and industry. Several important analytical results have been derived in the Stokes limit of colloids moving at low Reynolds numbers, including Stokes's drag on a spherical colloid, Einstein's viscosity of a dilute suspension of spherical colloids, and Jeffery's tumbling motion of an ellipsoidal colloid in a linear shear flow. ${ }^{1-6}$ Solving the mobility matrix and resulting motions of complexshaped rigid particles, however, typically requires a numerical approach. One way is to explicitly solve the flow around the body using direct numerical simulations, e.g., lattice Boltzmann simulations, but this is computationally demanding and becomes difficult at low Reynolds numbers. ${ }^{7,8}$

Building on the work by Oseen ${ }^{9}$ and Burgers ${ }^{10}$ for the flow field generated by a point force, Riseman and Kirkwood $^{11}$ derived translational and rotational diffusion tensors for rigid clusters of particles in the Stokesian limit. Bloomfield and co-workers ${ }^{12-14}$ and Goldstein, ${ }^{15}$ among others, extended this framework by incorporating the improved hydrodynamic interactions between two spheres derived by Rotne and Prager ${ }^{16}$ and Yamakawa. ${ }^{17}$ The result is a $(6 \times 6)$ mobility matrix relating the translational and rotational velocities of a colloidal body to the total force and torque acting on that body, taking into account the hydrodynamic interactions between the various parts of the body and implicitly solving the constraint forces and torques that rigidify the body. Several authors reported on codes to calculate this matrix, ${ }^{18,19}$

a)Electronic mail: w.k.denotter@utwente.nl while García de la Torre et al. ${ }^{20,21}$ made their Hydro++ code publicly available. The latter also combined a rotationally averaged weighted translational mobility matrix with a volume correction to predict intrinsic viscosities at zero shear rate. ${ }^{22,23}$ Brady and collaborators developed Stokesian Dynamics (SD) to simulate suspensions of (non-connected) spherical particles. ${ }^{24,25}$ In this scheme, the generalized velocities and forces are supplemented with stress and strain matrices to improve the accuracy of the hydrodynamic calculations, to simulate suspensions in linear flow fields, and to calculate viscosities of quiescent and flowing suspensions. Several authors hinted at and/or have worked out a generalized mobility matrix for arbitrarily shaped colloids including stress and strain, ${ }^{19,26-28}$ but a detailed description and thorough test of a generic method appears to be missing in the literature. The aim of the current paper is to describe the derivation of a generalized $(11 \times 11)$ grand mobility matrix, implemented in the publicly available Oseen11 code, and to compare simulation results obtained with this matrix against a number of analytical results for validation.

The generalized mobility matrix, obtained by the method outlined above or by the boundary element method, ${ }^{29,30}$ can be used to efficiently simulate the dynamics of the body. For a rigid object, the mobility matrix in the body-based frame remains constant; hence, it needs to be evaluated only once and its time-varying counterpart in the laboratory-based frame is readily obtained through rotation. The literature contains a number of simulations of this type, ${ }^{18,31,32}$ using quaternions to describe the orientation of the body relative to its hydrodynamic center. We show that this is a fortuitous choice. It is well known that the use of four quaternion coordinates removes the degeneracy encountered with three rotational coordinates, like the Euler angles or the components of a 
rotation vector. ${ }^{33}$ In the simulation of Brownian motion, however, the use of non-Cartesian coordinates gives rise to an additional metric-related term in the equations of motion. Furthermore, in the usual Itô representation of stochastic differential equations, the orientation-dependence of the spacebased mobility matrix also gives rise to an additional term in the equations of motion. ${ }^{34-36}$ Naess, Elgsaeter, and coworkers $^{37-41}$ derived expressions for these additional terms for simulations employing Euler angles and a rotation vector, respectively, assuming a block-diagonal mobility matrix. Ilie et $a .^{42}$ showed that the additional terms vanish identically when using quaternions, in combination with an exactly solved constraint to preserve the unit length of the quaternion vector, again assuming a block-diagonal mobility matrix. Their derivation is extended here to general mobility matrices, arriving at the convenient result that the additional terms cancel out when using quaternions to represent rotations around the mobility center. Alternatively, Makino and $\mathrm{Doi}^{29}$ employed a Fokker-Planck equation to derive an equation of motion using the nine elements of the rotation matrix as coordinates to describe the orientation of the object; their time evolution obeys the six orthonormality conditions to a rotation matrix only in the limit of vanishing time step, and consequently the calculated motion is subject to a gradual drift.

The outline of this paper is as follows: The derivations of the grand mobility matrix and the equations of motion are presented in Sec. II, with details referred to the appendices, culminating in the central expression of Eq. (19). Simulation results validating the algorithm are presented in Sec. III, where it is shown that translational and rotational Brownian dynamics, as well as the equilibrium Boltzmann distribution, are faithfully sampled for colloids in a quiescent fluid, while colloids in sheared fluids, in the absence of Brownian motion and external forces, correctly trace the analytical Jeffery orbits of ellipsoidal particles ${ }^{3}$ and spherical caps. ${ }^{43}$ We end with a brief summary of the main results.

\section{THEORY}

The constant body-based generalized mobility matrix of a rigid colloid fully describes the response of the colloid to external influences. In Subsections II A and II B, we derive this matrix for a body consisting of primary spherical particles and construct the corresponding Brownian Dynamics equation of motion.

\section{A. Mobility matrix}

Consider a collection of $N$ unconnected spherical particles suspended in an incompressible Newtonian viscous fluid. At low Reynolds numbers, the equations of motion of each particle are solved by balancing the potential-based force and torque on the particle with the hydrodynamic drag force and torque experienced by the particle, which in turn depend on the motions of all particles in the system. ${ }^{6}$ In the mobility representation, the translational velocity $\overline{\mathbf{v}}_{i}$ and rotational velocity $\overline{\boldsymbol{\omega}}_{i}$ of the $i$ th particle with position $\overline{\mathbf{x}}_{i}$ are related to the potentialbased forces $\overline{\mathbf{f}}_{j}$ and torques $\overline{\boldsymbol{\tau}}_{j}$ on all particles $j$, via the grand mobility matrix,

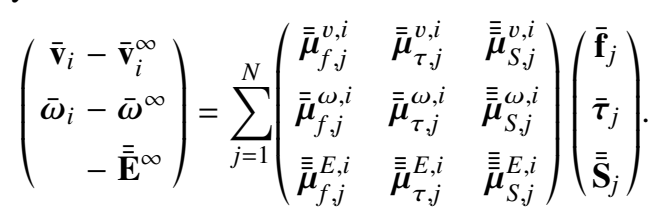

Here the balance is solved in the presence of a linear background flow field, $\overline{\mathbf{v}}_{i}^{\infty}=\overline{\mathbf{v}}^{\infty}\left(\overline{\mathbf{x}}_{i}\right)$, with

$$
\overline{\mathbf{v}}^{\infty}(\overline{\mathbf{x}})=\overline{\mathbf{v}}_{0}^{\infty}+\overline{\overline{\mathbf{E}}}^{\infty} \overline{\mathbf{x}}+\overline{\boldsymbol{\omega}}^{\infty} \times \overline{\mathbf{x}},
$$

where the strain rate $\overline{\overline{\mathbf{E}}}^{\infty}$ and angular velocity $\overline{\boldsymbol{\omega}}^{\infty}$ are uniform throughout the system and $\overline{\mathbf{v}}_{0}^{\infty}$ denotes the flow velocity at the origin of the laboratory coordinate system. The vector on the r.h.s. of Eq. (1) collects the force, torque, and stress, $\overline{\overline{\mathbf{S}}}_{j}$, transmitted by particle $j$ onto the fluid. The deformations of the particles are obtained by balancing the hydrodynamic stresses on the particle $\left(\right.$ i.e., $-\overline{\overline{\mathbf{S}}}_{j}$ ) and the potential-based stresses on the particle with the deformation stresses within the particle; for rigid particles, the latter reduce to Lagrange multipliers that balance any imposed stress at vanishing deformation of the particle. Approximate analytical expressions for the grand mobility matrix of two interacting spherical particles are available in the literature ${ }^{5,24}$ and summarized in Appendix A. Our objective in this subsection will be to derive, starting from Eq. (1) or from the equivalent (inverse) resistance problem in Eq. (6), a mobility matrix relating the translation, rotation, and stress of a rigid cluster of $N$ spherical particles to the total potential force, torque, and flow field. Two brief comments on the notation: the number of bars highlights the rank of a tensor, with each spatial index running over the usual three dimensions; for tensors with both a subscript and a superscript, the former denotes the intended multiplication partner and the latter denotes the resulting outcome.

The combination of various ranks in the grand mobility tensor disallows the use of standard numerical routines for square matrices. We therefore rewrite the strain rate of the flow field as a linear combination of nine $(3 \times 3)$ "basis matrices" $\overline{\overline{\mathbf{e}}}_{\kappa}^{E}$ and their "dual basis matrices" $\overline{\overline{\mathbf{e}}}_{E}^{\kappa}$,

$$
\begin{aligned}
\overline{\overline{\mathbf{E}}}^{\infty} & =\sum_{\kappa} \overline{\overline{\mathbf{e}}}_{\kappa}^{E} \mathcal{E}_{\kappa}^{\infty}, \\
\mathcal{E}_{\kappa}^{\infty} & =\overline{\overline{\mathbf{e}}}_{E}^{K}: \overline{\overline{\mathbf{E}}}^{\infty},
\end{aligned}
$$

where the set of coefficients $\mathcal{E}_{\kappa}^{\infty}$ constitute a column vector $\overline{\mathcal{E}}^{\infty}$ and the colon denotes a double contraction. Since in the context of hydrodynamics it proves convenient to use a non-orthogonal set of basis matrices, the basis matrices in the reverse transformation differ from those in the forward transformation; ${ }^{5}$ we refer to Appendix B for more details. Because the strain rate is defined as the symmetric part of the flow field gradient, for any divergence-free flow only five coefficients are required. If one is not interested in the hydrostatic pressure, the stresses on the particles-symmetric by definition-likewise reduce to a five-vector, $\overline{\mathcal{S}}_{j}$. We then arrive at

$$
\left(\begin{array}{c}
\overline{\mathbf{v}}_{i}-\overline{\mathbf{v}}_{i}^{\infty} \\
\overline{\boldsymbol{\omega}}_{i}-\overline{\boldsymbol{\omega}}^{\infty} \\
-\overline{\mathcal{E}}^{\infty}
\end{array}\right)=\sum_{j=1}^{N}\left(\begin{array}{ccc}
\overline{\overline{\boldsymbol{\mu}}}_{f, j}^{v, i} & \overline{\overline{\boldsymbol{\mu}}}_{\tau, j}^{v, i} & \overline{\overline{\boldsymbol{\mu}}}_{\mathcal{S}, j}^{v, i} \\
\overline{\overline{\boldsymbol{\mu}}}_{f, j}^{\omega, i} & \overline{\overline{\boldsymbol{\mu}}}_{\tau, j}^{\omega, i} & \overline{\overline{\boldsymbol{\mu}}}_{\mathcal{\mathcal { S }}, j}^{\omega, i} \\
\overline{\overline{\boldsymbol{\mu}}}_{f, j}^{\mathcal{E}} & \overline{\overline{\boldsymbol{\mu}}}_{\tau, j}^{\mathcal{E}, i} & \overline{\overline{\boldsymbol{\mu}}}_{\mathcal{S}, i}^{\mathcal{E}, i}
\end{array}\right)\left(\begin{array}{c}
\overline{\mathbf{f}}_{j} \\
\overline{\boldsymbol{\tau}}_{j} \\
\overline{\mathcal{S}}_{j}
\end{array}\right),
$$


where

$$
\begin{aligned}
& \overline{\overline{\boldsymbol{\mu}}}_{f, j}^{\mathcal{E}, i}=\overline{\overline{\mathbf{e}}}_{E}^{\mathcal{E}}: \overline{\overline{\boldsymbol{\mu}}}_{f, j}^{E, i}, \\
& \overline{\overline{\boldsymbol{\mu}}}_{\mathcal{S}, j}^{v, i}=\overline{\overline{\boldsymbol{\mu}}}_{S, j}^{v, i} \overline{\overline{\mathbf{e}}}_{\mathcal{S}} \text {, } \\
& \overline{\overline{\boldsymbol{\mu}}}_{\mathcal{S}, j}^{\mathcal{E}, i}=\overline{\overline{\mathbf{e}}}_{E}^{\mathcal{E}}: \overline{\overline{\overline{\boldsymbol{\mu}}}}_{S, j}^{E, i} \overline{\overline{\mathbf{e}}}_{\mathcal{S}}^{S},
\end{aligned}
$$

etcetera, where $\overline{\overline{\mathbf{e}}}_{E}^{\mathcal{E}}$ and $\overline{\overline{\mathbf{e}}}_{\mathcal{S}}^{S}$ denote the third-rank tensors combining the five basis matrices $\left\{\overline{\overline{\mathbf{e}}}_{E}^{\kappa}\right\}$ and $\left\{\overline{\overline{\mathbf{e}}}_{\kappa}^{S}\right\}$, respectively. Since we have recovered conventional vector-matrix products in Eq. (4), henceforth the bars will be omitted for notational convenience. Note that particles $i$ and $j$ are now coupled by an $(11 \times 11)$ matrix. Inversion of the $(11 N \times 11 N)$ grand mobility matrix yields the $(11 N \times 11 N)$ grand resistance matrix in the resistance representation of a collection of unconnected particles,

$$
\left(\begin{array}{c}
\mathbf{f}_{i} \\
\boldsymbol{\tau}_{i} \\
\mathcal{S}_{i}
\end{array}\right)=\sum_{j=1}^{N}\left(\begin{array}{ccc}
\boldsymbol{\xi}_{v, j}^{f, i} & \xi_{\omega, j}^{f, i} & \boldsymbol{\xi}_{\mathcal{E}, j}^{f, i} \\
\boldsymbol{\xi}_{v, j}^{\tau, i} & \boldsymbol{\xi}_{\omega, j}^{\tau, i} & \boldsymbol{\xi}_{\mathcal{E}, j}^{\tau, i} \\
\boldsymbol{\xi}_{v, j}^{\mathcal{S}, i} & \boldsymbol{\xi}_{\omega, j}^{\mathcal{S}, i} & \boldsymbol{\xi}_{\mathcal{E}, j}^{\mathcal{S}, i}
\end{array}\right)\left(\begin{array}{r}
\mathbf{v}_{j}-\mathbf{v}_{j}^{\infty} \\
\omega_{j}-\omega^{\infty} \\
-\mathcal{E}^{\infty}
\end{array}\right)
$$

The summation results on the r.h.s. can be interpreted as minus the hydrodynamic force, torque, and stress on particle $i$ at given linear and angular velocities of all particles $j$ in a given flow field. The hydrodynamic interactions remain unchanged when the particles are connected to form a rigid cluster. The particle velocities in a rigid cluster are related by

$$
\begin{aligned}
\mathbf{v}_{j} & =\mathbf{v}+\omega \times \mathbf{r}_{j}, \\
\omega_{j} & =\omega,
\end{aligned}
$$

where $\mathbf{r}_{j}=\mathbf{x}_{j}-\mathbf{x}$ denotes the vector connecting particle $j$ to a reference point on the cluster with spatial position $\mathbf{x}$, henceforth referred to as the position of the cluster; $\mathbf{v}=\dot{\mathbf{x}}$ represents the translational velocity of the cluster; and $\omega$ is its rotational velocity. The background flow velocity experienced by particle $j$, see Eq. (2), is then readily expressed as the background flow velocity experienced by the cluster, $\mathbf{v}^{\infty}(\mathbf{x})$, plus a linear transformation of $\mathbf{r}_{j}$.

Given the forces, torques, and stresses on the individual particles in a rigid cluster, the total force, torque, and stress on the cluster follow by the addition rules

$$
\begin{aligned}
\mathbf{f} & =\sum_{i=1}^{N} \mathbf{f}_{i}, \\
\boldsymbol{\tau} & =\sum_{i=1}^{N}\left(\boldsymbol{\tau}_{i}+\mathbf{r}_{i} \times \mathbf{f}_{i}\right), \\
\mathbf{S} & =\sum_{i=1}^{N}\left(\mathbf{S}_{i}+\mathbf{r}_{i} \otimes \mathbf{f}_{i}\right) .
\end{aligned}
$$

The first two of these equations are readily applied to the potential-based forces and torques on the particles, whereas in the third equation the stresses on the particles are still unknown. Applying these addition rules, in combination with Eq. (7), to the r.h.s. of Eq. $(6)$ yields the $(11 \times 11)$ grand resistance matrix of the cluster,

$$
\left(\begin{array}{c}
\mathbf{f} \\
\boldsymbol{\tau} \\
\mathcal{S}
\end{array}\right)=\left(\begin{array}{ccc}
\boldsymbol{\xi}_{v}^{f} & \xi_{\omega}^{f} & \boldsymbol{\xi}_{\mathcal{E}}^{f} \\
\boldsymbol{\xi}_{v}^{\tau} & \boldsymbol{\xi}_{\omega}^{\tau} & \boldsymbol{\xi}_{\mathcal{E}}^{\tau} \\
\boldsymbol{\xi}_{v}^{\mathcal{S}} & \boldsymbol{\xi}_{\omega}^{\mathcal{S}} & \boldsymbol{\xi}_{\mathcal{E}}^{\mathcal{S}}
\end{array}\right)\left(\begin{array}{c}
\mathbf{v}-\mathbf{v}^{\infty}(\mathbf{x}) \\
\omega-\omega^{\infty} \\
-\mathcal{E}^{\infty}
\end{array}\right),
$$

where the r.h.s. represents minus the generalized hydrodynamic forces on the cluster. Explicit expressions for the nine sub-matrices are provided in Appendix C. The generalized constraint forces acting between the particles in a rigid cluster are all internal to the cluster and therefore do not contribute to the dynamics of the cluster. Simulating the dynamics of the cluster requires evaluation of the velocities for given potentialbased generalized forces and a given background flow field. This is achieved by a partial inversion of the above equation, see Appendix D, to arrive at the grand mobility matrix of the cluster,

$$
\left(\begin{array}{l}
\mathbf{v}-\mathbf{v}^{\infty}(\mathbf{x}) \\
\omega-\omega^{\infty} \\
\mathcal{S}
\end{array}\right)=\left(\begin{array}{lll}
\boldsymbol{\mu}_{f}^{v} & \boldsymbol{\mu}_{\tau}^{v} & \boldsymbol{\mu}_{\mathcal{E}}^{v} \\
\boldsymbol{\mu}_{f}^{\omega} & \boldsymbol{\mu}_{\tau}^{\omega} & \boldsymbol{\mu}_{\mathcal{E}}^{\omega} \\
\boldsymbol{\mu}_{f}^{\mathcal{S}} & \boldsymbol{\mu}_{\tau}^{\mathcal{S}} & \boldsymbol{\mu}_{\mathcal{E}}^{\mathcal{S}}
\end{array}\right)\left(\begin{array}{c}
\mathbf{f} \\
\boldsymbol{\tau} \\
-\mathcal{E}^{\infty}
\end{array}\right)
$$

While the laboratory-based grand mobility matrix will vary with the orientation of the rigid cluster, the body-based matrix remains constant. Hence, in principle, the dynamics of the cluster can be simulated based on a single evaluation of the mobility matrix.

\section{B. Brownian Dynamics}

The laboratory positions of all particles in a rigid cluster can be expressed as

$$
\mathbf{x}_{i}=\mathbf{x}+\mathbf{A}_{(\mathrm{b})}^{(\mathrm{s})} \mathbf{r}_{i}
$$

where $\mathbf{x}$ denotes the space-based position of the reference point on the cluster that defines the origin of the body-based coordinate system, $\mathbf{r}_{i}$ represents the body-based coordinates of particle $i$, and $\mathbf{A}_{(\mathrm{b})}^{(\mathrm{s})}$ is the rotation matrix from the body frame (b) to the space frame (s). For numerical convenience, see Sec. I, the rotation will be described in terms of the four-vector quaternions $\mathbf{q}$, with a constraint of unit length, $|\mathbf{q}|=1$. Details on the rotation matrix, and the corresponding transformation matrices for angular velocities, can be found in Appendix E. The generalized mobility matrix to be used henceforth is the matrix evaluated in the body frame. The objective now is to obtain equations of motion for the position $\mathbf{x}$ and orientation $\mathbf{q}$ of the cluster.

For a particle in a quiescent fluid, experiencing a conservative potential $\Phi$, the Brownian equation of motion in generalized coordinates $\mathbf{Q}$ reads as ${ }^{34-36}$

$$
\begin{aligned}
\Delta \mathbf{Q}(t) & =\mathbf{Q}(t+\Delta t)-\mathbf{Q}(t) \\
& =-\boldsymbol{\mu}_{Q} \boldsymbol{\nabla}_{Q} \mathcal{A}_{Q} \Delta t+k_{B} T \nabla_{Q} \cdot \boldsymbol{\mu}_{Q} \Delta t+\delta \mathbf{Q},
\end{aligned}
$$

with $\Delta \mathbf{Q}(t)$ being the displacement at time $t$ over a time step $\Delta t$, mobility matrix $\mu_{Q}$, free energy $\mathcal{A}_{Q}$, Boltzmann's constant $k_{B}$, temperature $T$, and random Brownian displacements $\delta \mathbf{Q}$. The free energy is defined as

$$
\begin{aligned}
\mathcal{A}_{Q}(\mathbf{Q}) & =-k_{B} T \ln P_{Q}(\mathbf{Q}) \\
& =\Phi(\mathbf{Q})-\frac{1}{2} k_{B} T \ln g_{Q}(\mathbf{Q}),
\end{aligned}
$$


with the Boltzmann equilibrium probability distribution for a particle experiencing a potential $\Phi$ given by

$$
P_{Q}(\mathbf{Q}) d \mathbf{Q} \propto g_{Q}^{1 / 2}(\mathbf{Q}) \exp \left[-\frac{\Phi(\mathbf{Q})}{k_{B} T}\right] d \mathbf{Q},
$$

where the metric $g_{Q}$ measures (the square of) the volume in coordinate space of $d \mathbf{Q}$. The first term on the last line of Eq. (12) is akin to Eq. (10), with minus the gradient of the free energy providing the driving force and a multiplication by $\Delta t$ to turn velocities into displacements. In the third term on the last line of Eq. (12), the components of the Brownian displacement vector $\delta \mathbf{Q}$ have zero average, no memory of the preceding time steps (i.e., Markovian), and their correlations are related to the mobility matrix by the fluctuation-dissipation theorem, ${ }^{34-36}$

$$
\langle\delta \mathbf{Q} \otimes \delta \mathbf{Q}\rangle=2 k_{B} T \boldsymbol{\mu}_{Q} \Delta t,
$$

where the pointed brackets denote a canonical average. In the Itô representation, i.e., all terms on the last line of Eq. (12) are evaluated at time $t$, the equation of motion contains a divergence term (here, the second term on the r.h.s.) accounting for spatial variations of the mobility. Inclusion of this term, which appears natural when deriving the first order equation of motion from the second order Langevin equation, ${ }^{36}$ ensures that the proper equilibrium distribution is sampled by Brownian systems with coordinate-dependent mobilities, like the non-spherical colloids in this study. All aforementioned contributions are imperative in simulations using Euler angles or a rotation vector to represent the orientation of the cluster, along with Taylor expansions to solve weak singularities at specific orientations. ${ }^{37-41}$ Ilie $e t a l .{ }^{42}$ have recently shown that the equations of motion simplify considerably when using quaternions, which will be the approach followed and extended here.

For a $(6 \times 6)$ mobility matrix, and assuming translation and rotation to be decoupled, Ilie et al. ${ }^{42}$ derived the Brownian equations of motion for translation and rotation as

$$
\begin{aligned}
& \Delta \mathbf{x}=\mathbf{A}_{(\mathrm{b})}^{(\mathrm{s})}\left[\boldsymbol{\mu}_{f(\mathrm{~b})}^{v(\mathrm{~b})} \mathbf{A}_{(\mathrm{s})}^{(\mathrm{b})} \mathbf{f}^{(\mathrm{s})} \Delta t+\delta \mathbf{x}^{(\mathrm{b})}\right], \\
& \Delta \mathbf{q}=\mathbf{B}_{(\mathrm{b})}^{\dot{q}}\left[\boldsymbol{\mu}_{\tau(\mathrm{b})}^{\omega(\mathrm{b})} \mathbf{A}_{(\mathrm{s})}^{(\mathrm{b})} \boldsymbol{\tau}^{(\mathrm{s})} \Delta t+\delta \boldsymbol{\psi}^{(\mathrm{b})}\right]+\lambda \mathbf{q},
\end{aligned}
$$

respectively. Both equations have the same structure: the force (torque) in the space frame is rotated to the body frame by the inverse rotation matrix, $\mathbf{A}_{(\mathrm{s})}^{(\mathrm{b})}=\left(\mathbf{A}_{(\mathrm{b})}^{(\mathrm{s})}\right)^{-1}$, the balance with the hydrodynamic friction force (torque) is solved in the body frame, and the resulting (angular) velocity is rotated back to the space frame to update the coordinates. The $(4 \times 3)$ matrix $\mathbf{B}_{(\mathrm{b})}^{\dot{q}}$ combines the rotation of a body-based angular velocity to the space frame with the conversion to time derivatives of quaternions; see Appendix E. The stochastic translations $\delta \mathbf{x}^{(\mathrm{b})}$ and rotations $\delta \boldsymbol{\psi}^{(\mathrm{b})}$ are sampled in the body frame, each with zero mean and each separately obeying a fluctuation-dissipation theorem akin to Eq. (15). These random displacements are easily generated using two independent three-vectors $\boldsymbol{\Theta}_{x}$ and $\boldsymbol{\Theta}_{\psi}$ of uncorrelated memory-free random numbers with zero mean and unit variance, in combination with the symmetric square roots of the $(3 \times 3)$ mobility matrices,

$$
\begin{gathered}
\delta \mathbf{x}^{(\mathrm{b})}=\sqrt{2 k_{B} T \Delta t}\left(\boldsymbol{\mu}_{f(\mathrm{~b})}^{v(\mathrm{~b})}\right)^{1 / 2} \boldsymbol{\Theta}_{x}, \\
\delta \boldsymbol{\psi}^{(\mathrm{b})}=\sqrt{2 k_{B} T \Delta t}\left(\boldsymbol{\mu}_{\tau(\mathrm{b})}^{\omega(\mathrm{b})}\right)^{1 / 2} \boldsymbol{\Theta}_{\psi} .
\end{gathered}
$$

The metric and divergence terms in the generalized equation of motion vanish identically when simulating the translational motion in Cartesian coordinates. Neither term vanishes in the description of the rotational motion, but both turn out to be parallel to $\mathbf{q}$ and therefore they cancel against the constraint force along $\nabla_{q}|\mathbf{q}|=\mathbf{q}$ that preserves the unit length of the quaternion vector. ${ }^{42}$ The strength of the constraint force, i.e., the Lagrange multiplier $\lambda$, is solved from the condition of unit length,

$$
|\mathbf{q}(t+\Delta t)|=\left|\mathbf{q}^{\mathrm{u}}(t+\Delta t)+\lambda \mathbf{q}(t)\right|=1,
$$

where $\mathbf{q}^{\mathrm{u}}(t+\Delta t)$ denotes the quaternions following the unconstrained time step. One readily shows that this condition constitutes a quadratic equation in $\lambda$.

The above equations of motion can be generalized to $(6 \times 6)$ mobility matrices with coupled translational-rotational motion, i.e. matrices for which the cross-terms $\boldsymbol{\mu}_{\tau}^{v}$ and $\boldsymbol{\mu}_{f}^{\omega}=\left(\boldsymbol{\mu}_{\tau}^{v}\right)^{\mathrm{T}}$ are non-zero. We present the main results here, and refer the reader interested in the mathematical details of the derivation to Appendix F. The equations of motion including Brownian noise take their simplest form when $\boldsymbol{\mu}_{f}^{\omega}=\boldsymbol{\mu}_{\tau}^{v}$, which occurs when the origin of the body-based coordinate system coincides with the hydrodynamic center of the cluster. We will henceforth adhere to this convenient choice and identify $\mathbf{x}$ with the space-based position of the hydrodynamic center-note that the equations of motion in the absence of Brownian noise will be of the same form for any chosen reference point. Equations to locate this center, and to subsequently "shift" the cluster mobility matrix to this center without repeating the calculation of Sec. II A, are included in Appendix G. Upon adding the displacements due to the linear flow field, the equations of motion read as

$$
\begin{aligned}
& \left(\begin{array}{c}
\Delta \mathbf{x} \\
\Delta \mathbf{q}
\end{array}\right)=\left(\begin{array}{cc}
\mathbf{A}_{(\mathrm{b})}^{(\mathrm{s})} & \mathbf{0} \\
\mathbf{0} & \mathbf{B}_{(\mathrm{b})}^{\dot{q}}
\end{array}\right)\left[\left(\begin{array}{ccc}
\boldsymbol{\mu}_{f}^{v} & \boldsymbol{\mu}_{\tau}^{v} & \boldsymbol{\mu}_{\mathcal{E}}^{v} \\
\boldsymbol{\mu}_{f}^{\omega} & \boldsymbol{\mu}_{\tau}^{\omega} & \boldsymbol{\mu}_{\mathcal{E}}^{\omega}
\end{array}\right)_{(\mathrm{b})}^{(\mathrm{b})}\left(\begin{array}{c}
\mathbf{A}_{(\mathrm{s})}^{(\mathrm{b})} \mathbf{f}^{(\mathrm{s})} \\
\mathbf{A}_{(\mathrm{s})}^{(\mathrm{b})} \boldsymbol{\tau}^{(\mathrm{s})} \\
-\mathcal{E}^{\infty(\mathrm{b})}
\end{array}\right) \Delta t+\left(\begin{array}{c}
\delta \mathbf{x}^{(\mathrm{b})} \\
\delta \boldsymbol{\psi}^{(\mathrm{b})}
\end{array}\right)\right] \\
& +\left(\begin{array}{c}
\mathbf{v}^{\infty(\mathrm{s})} \\
\mathbf{B}_{(\mathrm{s})}^{\dot{q}}
\end{array} \omega^{\infty(\mathrm{s})}\right) \Delta t+\left(\begin{array}{c}
\mathbf{0} \\
\lambda \mathbf{q}
\end{array}\right) .
\end{aligned}
$$

In the first term between square brackets, the forces and torques in the space frame are converted to the body frame by a single rotation, while the corresponding conversion of the strain rate involves two rotations followed by a reduction to five-vector,

$$
\mathcal{E}_{\kappa}^{\infty(\mathrm{b})}=\mathbf{e}_{E}^{K}:\left[\mathbf{A}_{(\mathrm{s})}^{(\mathrm{b})} \mathbf{E}^{\infty(\mathrm{s})} \mathbf{A}_{(\mathrm{b})}^{(\mathrm{s})}\right] .
$$

The generalized velocities are solved from a force balance in the body frame, converted back to the space frame and multiplied by the time step to obtain a displacement. In the second term between square brackets, the stochastic displacements are calculated using the symmetric square root matrix of the symmetric $(6 \times 6)$ top-left sub-matrix of the grand mobility matrix, in combination with a random six-vector $\boldsymbol{\Theta}_{x \psi}$ whose 
elements have zero mean, unit variance and are devoid of correlations,

$$
\left(\begin{array}{c}
\delta \mathbf{x}^{(\mathrm{b})} \\
\delta \boldsymbol{\psi}^{(\mathrm{b})}
\end{array}\right)=\sqrt{2 k_{B} T \Delta t}\left[\left(\begin{array}{cc}
\boldsymbol{\mu}_{f}^{v} & \boldsymbol{\mu}_{\tau}^{v} \\
\boldsymbol{\mu}_{f}^{\omega} & \boldsymbol{\mu}_{\tau}^{\omega}
\end{array}\right)_{(\mathrm{b})}^{(\mathrm{b})}\right]^{1 / 2} \boldsymbol{\Theta}_{x \psi} .
$$

The penultimate term to Eq. (19) describes the particle being carried along and rotated by the flow field. The final term represents the constraint introduced to preserve the unit length of the quaternion vector, which is solved using Eq. (18).

In the absence of Brownian motion, i.e., for $T=0$, the mobility matrix gives the stresses exerted by the body on the fluid, expressed in the body frame, as

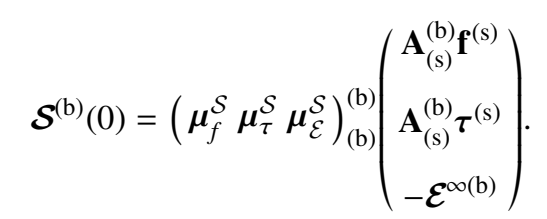

Conversion to a stress tensor in the space frame is achieved by a vector to tensor transformation, followed by two rotations,

$$
\mathbf{S}^{(\mathrm{s})}=\mathbf{A}_{(\mathrm{b})}^{(\mathrm{s})}\left[\sum_{K} \mathbf{e}_{\kappa}^{S} \mathcal{S}_{K}^{(\mathrm{b})}\right] \mathbf{A}_{(\mathrm{s})}^{(\mathrm{b})} \cdot
$$

We note that for a non-Brownian cluster the stresses are linearly related to the velocities of the body and hence to the displacements over a time step $\Delta t$. Extending this relation to the Brownian case, i.e., assuming that Brownian forces and external forces that generate identical displacements will also induce identical stresses, one arrives at

$\mathcal{S}^{(\mathrm{b})}(T)=\mathcal{S}^{(\mathrm{b})}(0)+\sqrt{\frac{2 k_{B} T}{\Delta t}}\left(\boldsymbol{\mu}_{f}^{\mathcal{S}} \boldsymbol{\mu}_{\tau}^{\mathcal{S}}\right)_{(\mathrm{b})}^{(\mathrm{b})}\left[\left(\begin{array}{cc}\boldsymbol{\mu}_{f}^{v} & \boldsymbol{\mu}_{\tau}^{v} \\ \boldsymbol{\mu}_{f}^{\omega} & \boldsymbol{\mu}_{\tau}^{\omega}\end{array}\right)_{(\mathrm{b})}^{(\mathrm{b})}\right]^{-1 / 2} \boldsymbol{\Theta}_{x \psi}$.

The prefactor $\Delta t^{-1 / 2}$ in the last term appears because the stress represents the time-averaged stress over the time step $\Delta t$, while the standard deviation of the Brownian force, i.e., a series of uncorrelated kicks by the solvent molecules, increases as $\Delta t^{1 / 2}$. Since for any given configuration the last term in the above expression averages to zero, we conclude that in the stationary state Brownian motion affects the stress only indirectly, i.e., by its impact on the distribution being sampled.

\section{SIMULATION RESULTS}

To validate the proposed algorithm, the various contributions to the equations of motion were tested, on an individual basis and/or in combinations, by comparison against known analytical solutions. The units used in the simulation are $\epsilon$ for energy, $\sigma$ for distance, and $\tau$ for time.

\section{A. Brownian motion}

To test the Brownian contributions to the equations of motion, we consider an anisotropic particle with the bodybased diagonal translational and rotational mobility matrices having the diagonal values $(5,7,9) \sigma^{2}(\tau \epsilon)^{-1}$ and $(0.5,4,10)$ $(\tau \epsilon)^{-1}$, respectively. The temperature is set at $k_{B} T=1 \epsilon$, and the time step is set at $\Delta t=1 \cdot 10^{-4} \tau$.
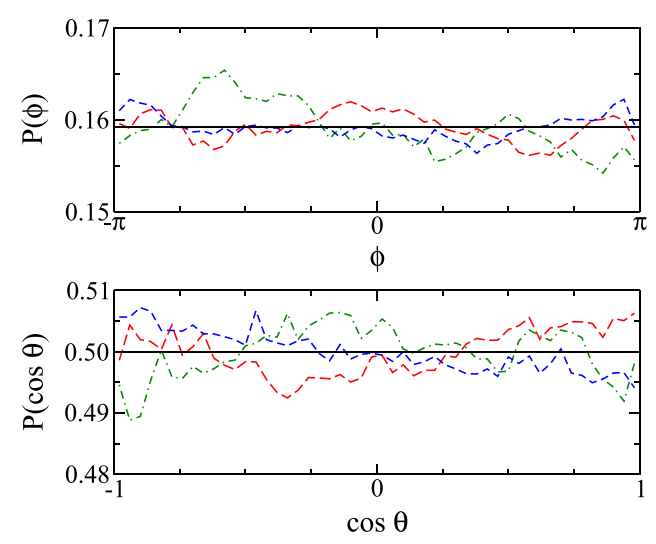

FIG. 1. Probability distributions of the azimuthal angles $\phi$ and (the cosines of) the polar angles $\theta$, relative to the space frame, of the three body-fixed eigenvectors of the mobility matrix of an anisotropic particle performing Brownian rotational diffusion. The solid lines represent the theoretical predictions.

The orientational probability distribution of the particle, in an isotropic environment, is analysed by sampling the orientations of the three body-fixed eigenvectors $\hat{\mathbf{u}}_{i}^{(\mathrm{b})}$ of the mobility matrix, as seen from the space-fixed frame, $\hat{\mathbf{u}}_{i}^{(\mathrm{s})}(t)=\mathbf{A}_{(\mathrm{b})}^{(\mathrm{s})}(t) \hat{\mathbf{u}}_{i}^{(\mathrm{b})}$. The probability distributions of the polar angles $\theta_{i}$ and azimuthal angles $\phi_{i}$ for the three body-based basis vectors, see Fig. 1, agree well with the expected isotropic distributions.

The dynamic properties are analysed by measuring the diffusional behaviour of the particle. In an isotropic medium, with all particle orientations equally likely, the average translational diffusion coefficient along any space-fixed direction is given by $D^{(\mathrm{s})}=\frac{1}{3} k_{B} T \operatorname{Tr}\left(\boldsymbol{\mu}_{f(\mathrm{~b})}^{v(\mathrm{~b})}\right)$, where $\operatorname{Tr}$ denotes the trace. The simulated time-dependent mean square displacements along the three space-fixed Cartesian axes overlay the theoretically expected curves; see Fig. 2.

The rotational diffusion is characterized by calculating the time correlation of a body-fixed vector $\hat{\mathbf{u}}^{(\text {b) }}$, as seen from the space-fixed frame,

$$
G_{\hat{\mathbf{u}}^{(\mathrm{b})}}(t)=\frac{3}{2}\left\langle\left(\hat{\mathbf{u}}^{(\mathrm{s})}(t) \cdot \hat{\mathbf{u}}^{(\mathrm{s})}(0)\right)^{2}\right\rangle-\frac{1}{2},
$$

with $\hat{\mathbf{u}}^{(\mathrm{s})}(t)=\mathbf{A}_{(\mathrm{b})}^{(\mathrm{s})}(t) \hat{\mathbf{u}}^{(\mathrm{b})}$. The time-correlation function has been solved theoretically as a sum of five exponentials, ${ }^{29,44}$

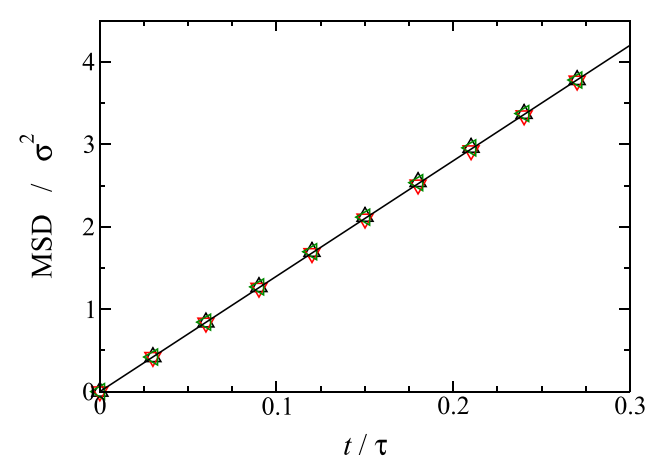

FIG. 2. Mean square displacements along the three space-fixed axes (markers) of a Brownian diffusing anisotropic particle, along with the theoretical prediction (line). 


$$
G_{\hat{\mathbf{u}}^{(\mathrm{b})}}(t)=\sum_{i=1}^{5} a_{i} e^{-t / \tau_{i}},
$$

where the five amplitudes $a_{i}$ and relaxation times $\tau_{i}$ are functions of the three rotational diffusion coefficients of the body and the three body-fixed components of the vector $\hat{\mathbf{u}}^{(\mathrm{b})}$. Figure 3 shows good agreement between the time-correlation functions from the simulations and the theoretical curves.

The aforementioned tests were repeated with an asymmetric body, a helix of 81 nearly touching particles forming a single $360^{\circ}$ turn with a radius of $12.5 \sigma$ and a height of $25 \sigma$. The simulation results (data not shown) are again in good agreement with theory. Taken together, these tests validate the inclusion of the stochastic terms in the equations of motion.

\section{B. Potentials}

Consider a particle with two point charges $q_{i}= \pm q$ placed at distances $\pm \frac{1}{2} d$ from the center of the particle along a direction $\hat{\mathbf{u}}^{(\mathrm{b})}$, creating a constant dipole moment $\mathbf{p}^{(\mathrm{b})}=q d \hat{\mathbf{u}}^{(\mathrm{b})}$ in the body frame and a variable dipole moment $\mathbf{p}^{(\mathrm{s})}(t)=\mathbf{A}_{(\mathrm{b})}^{(\mathrm{s})}(t) \mathbf{p}^{(\mathrm{b})}$ in the space frame. In the presence of an external electric field $\mathbf{E}^{(\mathrm{s})}$, the charges experience forces $\mathbf{f}_{i}^{(\mathrm{s})}=q_{i} \mathbf{E}^{(\mathrm{s})}$. The net force acting on the particle is zero, while the two forces induce a torque $\tau^{(\mathrm{s})}=\mathbf{p}^{(\mathrm{s})} \times \mathbf{E}^{(\mathrm{s})}$ that tends to align the dipole with the field. In the presence of thermal noise, the angle $\theta$ between the electric field and the dipole moment should obey the Boltzmann distribution $P(\cos \theta)=Z^{-1} \exp (\beta p E \cos \theta)$, where $Z$ is the normalizing configuration integral, irrespective of the mobility matrix. A particle with the aforementioned mobilities was simulated both using a single torque acting on a body-fixed dipole vector and using two forces acting on two body-fixed charges, obtaining good agreement with theory in both cases; see Fig. 4. These tests validate the implementation of the conservative and stochastic terms in the equation of motion.

\section{Flow fields}

The flow-induced particle dynamics were tested in the absence of conservative and stochastic terms to allow comparison with analytical expressions in the literature. Simulations of spheres, ellipsoids, and hemispherical caps were performed

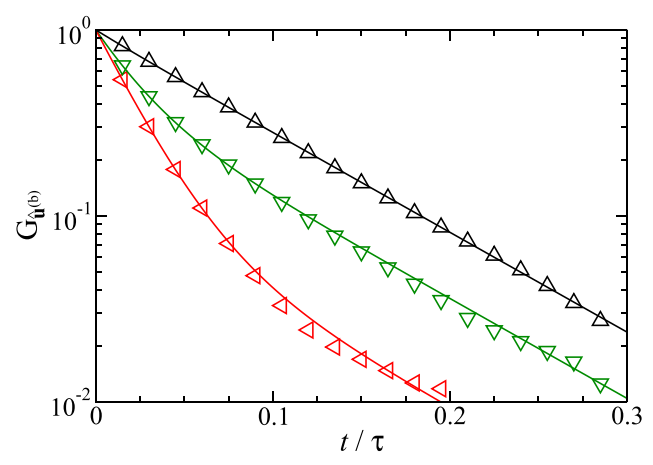

FIG. 3. Time-correlation functions of the three body-fixed eigenvectors of the rotational mobility matrix, as seen in the lab frame, compared with theoretical prediction (lines).

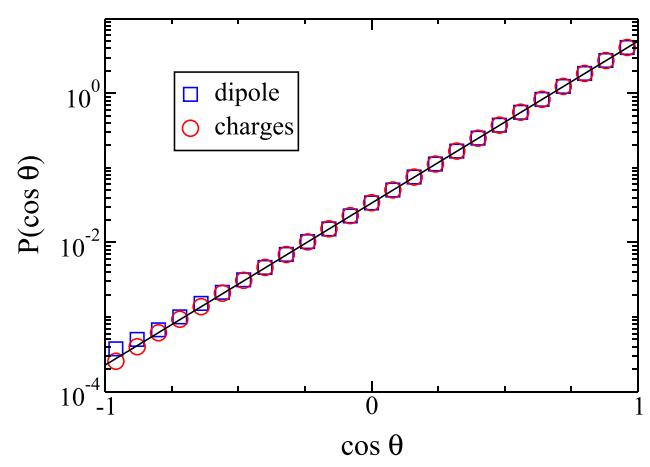

FIG. 4. Probability distribution of the angle $\theta$ between the dipole moment of a particle and an external uniform electric field, for $\beta p E=5$, and the corresponding theoretical result (line).

at a shear rate of $\dot{\gamma}=0.01 \tau^{-1}$ in a solvent of viscosity $\eta_{\mathrm{s}}=(6 \pi)^{-1} \epsilon \tau / \sigma^{3}$, using a time step of $\Delta t=0.01 \tau$.

\section{Spheres}

The simplest body is a rigid sphere. Due to its symmetries, the mobility matrix is block diagonal and the sphere merely translates and rotates along with the background fluid, $\mathbf{v}=\mathbf{v}^{\infty}(\mathbf{x})$ and $\omega=\omega^{\infty}$. The presence of a rigid body induces stress in the fluid, which is evaluated by Eqs. (22) and (23). For ease of comparison, we convert all calculated stresses into Einstein coefficients,

$$
B_{\alpha \beta}=\frac{1}{\eta_{\mathrm{s}} V_{\mathrm{c}}} \frac{S_{\alpha \beta}^{(\mathrm{s})}}{E_{\alpha \beta}^{\infty(\mathrm{s})}},
$$

with $V_{\mathrm{c}}$ being the volume of the colloid. For a rigid sphere, all elements of $\mathbf{B}$ should be equal to 5/2. ${ }^{2,6}$ When simulating the sphere as a single primary particle in a shear flow with shear rate $\dot{\gamma}$, hence $\mathbf{v}^{\infty}(\mathbf{x})=\dot{\gamma} y \hat{\mathbf{e}}_{x}^{(\mathrm{s})}$, the two non-zero elements of the stress and strain matrices, $x y$ and $y x$, both yield Einstein coefficients that approach the theoretical value to within numerical accuracy. Besides shear flow, the algorithm also permits planar, uniaxial, and biaxial extensional flows. In all cases, the nonzero elements in the stress and strain tensor yield an Einstein coefficient of $5 / 2$, in agreement with theory.

The simulation of more complex bodies requires the construction of a rigid shell of primary spheres such that the collective outer envelope of the primary particles closely approaches the outer surface of the desired body. To assess the validity of this approach, a sphere was modeled as a collection of $N=2082$ beads of radius $a=1 \sigma$ forming a hollow shell. The beads were placed on the vertices of a geodesic spherical dome, created with the DistMesh routine ${ }^{45}$ in matlab, ${ }^{46}$ and subsequently shifted along the radial direction to place all bead centers at equal distance $R=32 \sigma$ from the sphere's center. Table I collects the Einstein coefficients obtained from the simulations, with standard deviations resulting from the time-varying orientation of the near-spherical body relative to the shear flow (the sphere rotates with an angular velocity $\omega=\omega^{\infty(\mathrm{s})}=\dot{\gamma} / 2$ around the vorticity direction). Since the body's surface is not uniquely defined, the volume entering Eq. (27) was calculated based on i) a sphere with radius $R$ matching the distance between bead centers and sphere center, ii) the former volume augmented with the collective volume 
TABLE I. Einstein coefficients $B_{x y}$ for a hollow sphere of 2082 primary particles in an $x y$ shear flow, calculated using Eq. (27), assuming the three definitions of the sphere's volume discussed in the main text. The last line shows the effective radius corresponding to the theoretical value $B_{x y}=2.5$. The standard deviations result from the rotating spherical shell not being perfectly spherical.

\begin{tabular}{lcc}
\hline \hline Surface & $R$ & $B_{x y}$ \\
\hline Centers & 32 & $2.6488 \pm 1 \cdot 10^{-4}$ \\
Centers and bumps & $32-33$ & $2.5672 \pm 1 \cdot 10^{-4}$ \\
Circumscribed & 33 & $2.4152 \pm 1 \cdot 10^{-4}$ \\
Effective sphere & $32.6226 \pm 1 \cdot 10^{-4}$ & 2.5 \\
\hline \hline
\end{tabular}

$\frac{2}{3} N \pi a^{3}$ of the hemispherical bumps decorating the former surface, and iii) the volume of the circumscribed sphere of radius $R+a$. The second option yields a $B_{x y}$ nearest the theoretical Einstein coefficient of 2.5. Assuming the latter value as given, one may also invert the calculation to determine the effective radius of the body, as in the last line of Table I. Comparing the calculated translation and rotation diffusion coefficients with their well-known theoretical counterparts, $D_{t}=k_{B} T /\left(6 \pi \eta_{\mathrm{s}} R\right)$ and $D_{r}=k_{B} T /\left(8 \pi \eta_{\mathrm{s}} R^{3}\right)$, yields relative errors for translation diffusion of $-2.1 \%, 1.2 \%$, and $-0.03 \%$ and for rotation diffusion of $-5.8 \%, 3.3 \%$, and $-0.02 \%$, when using sphere radii based on the centers of the primary particles, the circumscribed sphere, and the effective sphere, respectively.

When modeling a body as a shell of nearly touching identical primary particles, the numerical results depend on the number of particles $N$ as well as on the highest order $r_{i j}^{-n}$ included in the expansion of the hydrodynamic interactions between pairs of particles, i.e., the series in Eq. (A5). Figure 5 collects results for the translation diffusion coefficient, the rotation diffusion coefficient, and the Einstein coefficient of a spherical body for three values of $N$ and five values of $n$. To enable comparison, all coefficients are converted into effective radii using the aforementioned expressions and divided by the radius of the sphere containing the centers of the particles. For the $N=8$ cubic representation frequently used in the literature, ${ }^{23,47}$ the rescaled radii show significant

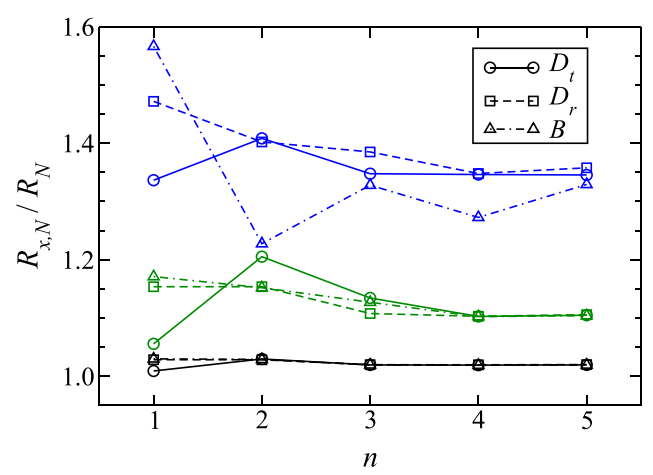

FIG. 5. Impact of truncating the hydrodynamic pair interactions at order $n$ in the reciprocal inter-particle distance, $r_{i j}^{-1}$. For ease of comparison, the calculated translation diffusion coefficient $D_{t}$, rotation diffusion coefficient $D_{r}$, and Einstein coefficient $B$ for spherical shells of $N=8$ (blue), 102 (green), and 2082 (black) primary particles have been converted to effective sphere radii $R_{x, N}$ using their well-known theoretical expressions and divided by the radius $R_{N}$ of the sphere through the centers of the primary particles. differences that decrease in a non-monotonic way with increasing order $n$. With an increasing number of particles, the three effective radii tend to be in better agreement, while the radius of the body increases. Consequently, at $N=102$, the standard deviation of the six effective radii for $n=4$ and 5 has reduced to $\sim 1 \%$ o of the average, while for $N=2082$ the nine effective radii for $n \geq 3$ agree to within $\sim 0.3 \%$ of the average. Hence, employing more primary particles appears as the more appealing method to attaining an accurate description of a complex body, rather than extending the hydrodynamic pair interaction to higher orders in the distance.

\section{Ellipsoids}

The second body shape considered is a prolate ellipsoid of revolution, also known as a prolate spheroid, which has been studied extensively in the past. ${ }^{3,10,48}$ Because of the reduced symmetry relative to the sphere, the block diagonal grand mobility matrix of the sphere becomes augmented by off-diagonal blocks coupling flow and rotation, i.e., $\boldsymbol{\mu}_{\mathcal{E}}^{\omega}$ and $\boldsymbol{\mu}_{\tau}^{\mathcal{S}}$. A characteristic feature of an ellipsoid in shear flow is its non-uniform tumbling motion, see Fig. 6, commonly referred to as Jeffery orbits, while the center of the ellipsoid translates uniformly with the flow. Jeffery ${ }^{3}$ derived that ellipsoidal bodies trace periodic orbits with the in-plane rotation angle of the long axis evolving as

$$
\tan \phi(t)=p \tan \frac{\dot{\gamma} p t}{p^{2}+1},
$$

where $p=L / D$ denotes the aspect ratio of the ellipsoid, with $L$ and $D$ being the lengths of the long and short axes, respectively. Due to the asymmetric shape, the stress induced on the fluid varies with the orientation of the ellipsoid. Jeffery also evaluated the excess work when shearing a fluid containing an ellipsoid of volume $V_{\mathrm{c}}$, which for an ellipsoid tumbling at $\theta=\pi / 2$ translates into an orientation dependent Einstein

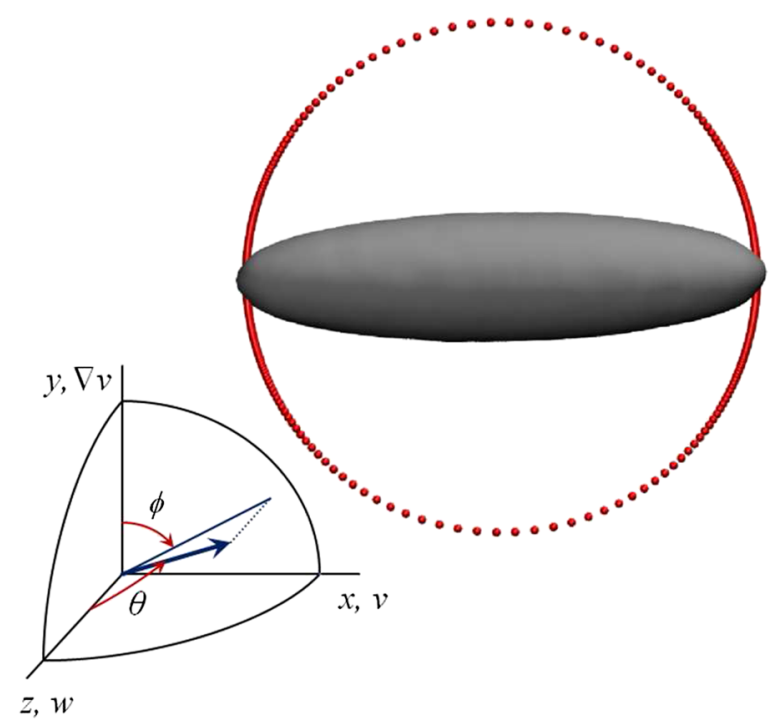

FIG. 6. Snapshot of a prolate ellipsoid with aspect ratio $p=5$, periodically tumbling clockwise around the $z$ axis in a linear shear flow $\mathbf{v}^{\infty}(\mathbf{x})=\dot{\gamma} y \hat{\mathbf{e}}_{x}^{(\mathrm{s})}$. The red dots, marking the position of one tip at equal time intervals, illustrate the non-linear angular velocity $\dot{\phi}$ of the particle's Jeffery orbit, with $\theta=\pi / 2$ throughout. 
coefficient,

$$
B_{x y}(\phi)=\frac{4}{3 V_{p}}\left\{F \sin ^{2} 2 \phi+G\right\},
$$

where $F$ and $G$ are functions of the aspect ratio.

In the simulations, ellipsoids with an aspect ratio $p=5$ are modelled as hollow shells composed of spherical beads. The positions of the primary particles $\mathbf{x}_{j}$ are generated by triangulation of the ellipsoid's surface, again using DistMesh. Each particle is then displaced along the vector $\mathbf{r}_{j}=\mathbf{x}_{j}-\mathbf{x}$, with $\mathbf{x}$ being the center of the ellipsoid, to ensure that the outer surfaces of all particles touch the circumscribed ellipsoid of a desired aspect ratio. The body's grand resistance and mobility matrices are calculated using the method outlined in Sec. II A, taking the primary particle's radius $a$ as (slightly less than) half the minimum distance between two adjacent vertices. Analysis of the nine unique non-zero matrix elements of the resistance matrix shows that their relative differences from their theoretical values ${ }^{5}$ scale approximately linearly with $N^{-1}$; see Fig. 7 . The deviations from the fitted lines are correlated, especially those of the translational and rotational resistances, suggesting that the accuracy in describing an ellipsoidal body depends not only directly on the number of beads but also indirectly via the $N$-dependent triangulation of the surface.

Simulations of the ellipsoidal bodies in simple shear flows yield periodic orbits, with the long axis rotating in a nonuniform fashion around the vorticity direction, while simultaneously the short axes rotate around the long axis. The magnitudes of these two motions vary with the angle $\theta$, culminating in a pure tumbling motion for $\theta=\pi / 2$ and a pure rolling motion for $\theta=0$; these are also the only two values at which $\theta$ remains constant, while all other orientations result in a coupling between $\theta$ and $\phi$ in excellent agreement with Jeffery's theory. ${ }^{3}$ As an example, Fig. 8 shows the pure tumbling motions of an ellipsoidal body when simulated using three differing numbers of primary particles, as well as the theoretical prediction, with all four orbits re-scaled by their respective periods $\tau_{N}$ for the ease of comparison. The angular velocity $\dot{\phi}$ periodically varies between near-zero, when the long axis is flow-aligned, and $\dot{\phi}=\dot{\gamma}=2 \omega^{\infty(\mathrm{s})}$, when the particle is oriented along the gradient direction. By contrast,

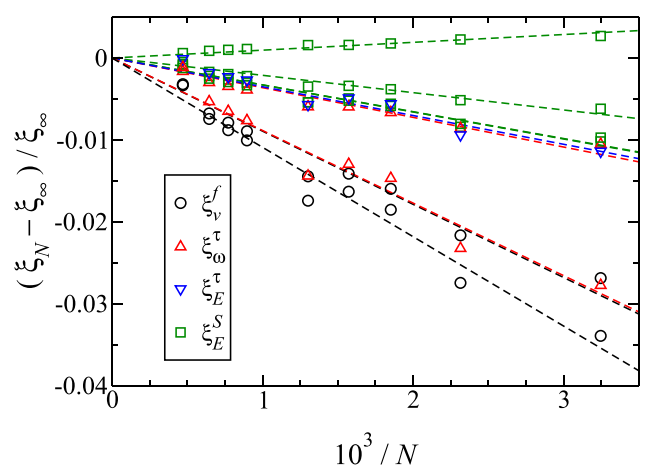

FIG. 7. Deviations of the nine unique non-zero components of the resistance matrix of a prolate ellipsoid, relative to their theoretical values (indicated by a subscript $\infty$ ), plotted against the reciprocal number of primary particles in the representation of the ellipsoid. The markers denote the corresponding blocks of the resistance matrix; the straight lines are fitted by imposing a vanishing intercept.

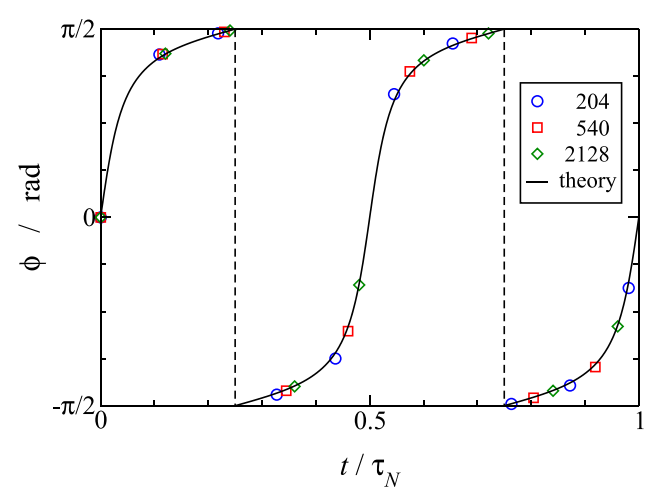

FIG. 8. Periodic tumbling of an ellipsoid in a shear flow, with $\phi$ being the angle between the long axis and the velocity gradient direction (mapped to the range $[-\pi / 2, \pi / 2]$ ), with a long axis permanently perpendicular to the vorticity direction, $\theta=\pi / 2$. The markers, with the numbers of primary particles $N$ denoted in the legend, are equally spaced in time and ought ideally have coalesced for the three representations of the same ellipsoidal body. Agreement with Jeffery's theory (solid line) is obtained by rescaling the orbits with their respective periods $\tau_{N}$; see also Fig. 9 .

the center of the body translates at a uniform velocity. The periods of a dozen realizations of the same ellipsoidal body converge with increasing $N$ to the theoretical limit, $\tau_{\infty}$; see Fig. 9. Over the explored range of $90 \leq N \leq 2128$, the periods are well described by a power law, $\left(\tau_{N}-\tau_{\infty}\right) / \tau_{\infty} \approx 6.6 N^{-0.73}$.

The tumbling motion of the body causes the Einstein coefficient $B_{x y}$ to vary periodically too. Simulation results, for three representations of a $p=5$ ellipsoidal body, yield the same characteristic curve as the theoretical prediction; ${ }^{3,49}$ see Fig. 10. The coefficient shows broad minima for nearly flow-aligned orientations, with $B_{x y}$ slightly undershooting the value of 2.5 for a sphere, alternating with maxima when the particle is at increased angles to the flow velocity; the narrow dip in this maximum coincides with the particle briefly reaching an angular velocity matching the shear rate of the imposed flow. The largest difference between numerical and theoretical values, both in relative and in absolute terms, is found at the minima of the curves. With an increasing number of primary particles, the simulation results increase to their theoretical values over the entire time range. The effect of the volume evaluation on the Einstein coefficient is explored in Fig. 11. Like for the

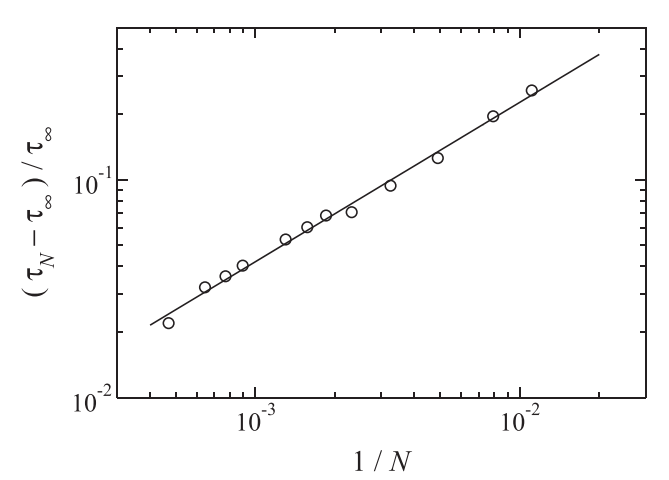

FIG. 9. Relative deviation in the tumbling periods $\tau_{N}$ of $p=5$ ellipsoidal bodies, modelled as rigid shells of $N$ spherical primary particles, in a shear flow. The limiting value, $\tau_{\infty}$, is the period derived by Jeffery. The line is a power-law fit. 


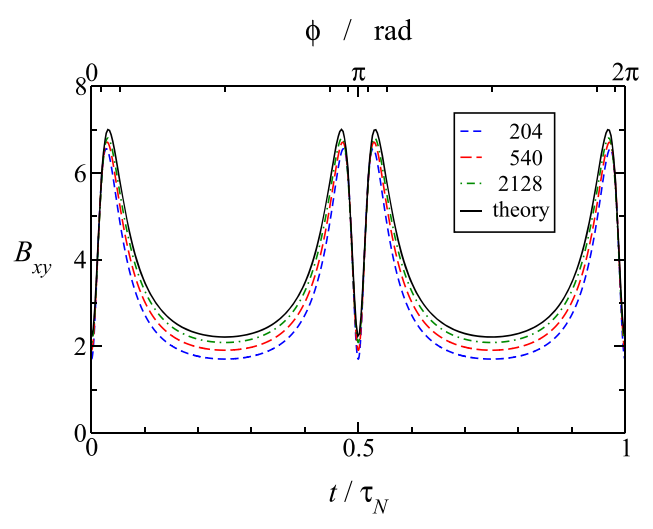

FIG. 10. Time evolution of the Einstein coefficient $B_{x y}$ for an ellipsoidal body in a shear flow, as simulated using differing numbers of primary particles and as derived from Jeffery's theory. The volume entering Eq. (27) is that of the ellipsoid enveloping the primary particles [see also Fig. 11]. On the top axis, the interval between successive ticks corresponds to a rotation $\Delta \phi=\pi / 6$.

spherical body, the volume is calculated based on (i) the ellipsoidal body formed by the centers of the primary particles, (ii) the increment hereof by including the collective volume of the half-spheres protruding from this body, and (iii) the ellipsoidal body that circumscribes the primary particles. The analytical result is bracketed by the latter two volumes over the entire time range, suggesting that the effective volume of the simulated body lies between these two limits. When using a number of primary particles in the low hundreds, however, the Einstein coefficients calculated using these two limiting volumes no longer bracket the theoretical curves over the entire range (data not shown), indicating that the compound body ceases to accurately describe the desired ellipsoidal shape for low $N$.

\section{Hemi-spherical caps}

A spherical cap, i.e., a fragment of a spherical shell with radius $R$ and top angle $\Theta$, also performs periodic orbits in a linear shear flow; see Fig. 12. We again focus on orbits with the rotational symmetry axis of the body at a constant angle of $\theta=\pi / 2$ to the vorticity direction. As shown by Dorrepaal, ${ }^{43}$ the rotational motion of the spherical cap is similar to that of an ellipsoid,

$$
\tan \phi(t)=\rho \tan \frac{2 \pi t}{\tau}
$$

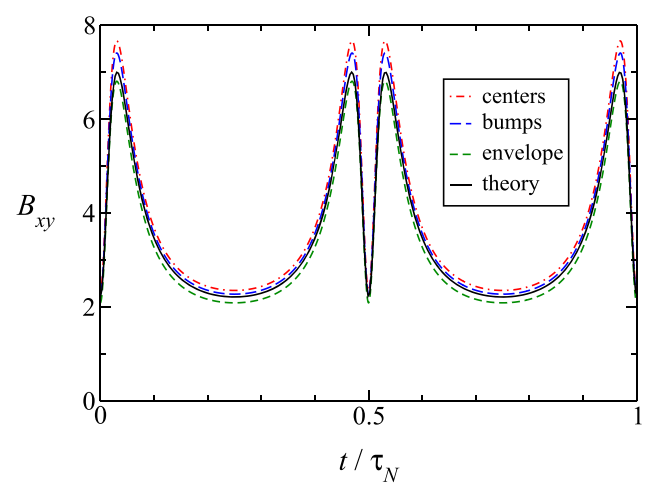

FIG. 11. Influence of the volume calculation on the Einstein coefficient of an ellipsoidal body approximated by a shell of 2128 primary particles; see Eq. (27).

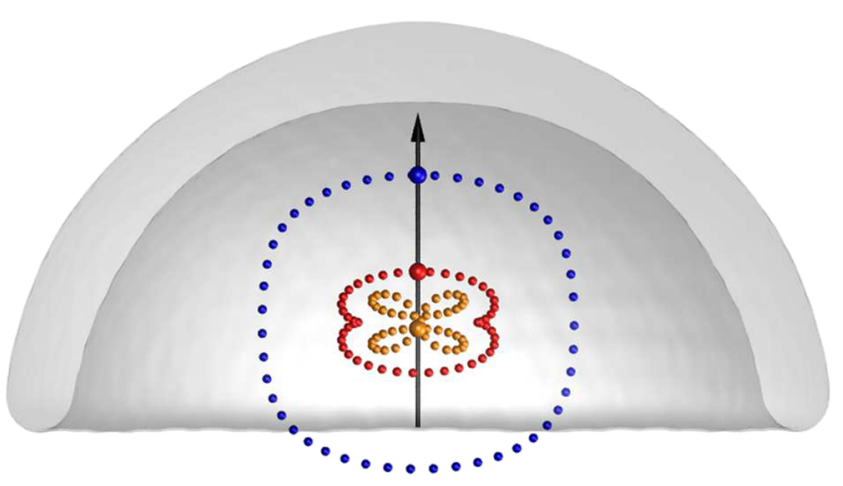

FIG. 12. Cross section of a hemi-spherical cap through its symmetry axis. The coloured dots denote the positions, equally spaced in time, of three points on the cap's symmetry axis (arrow starting at the center of the cap) during the combined rotational and translational motion of this body in a linear shear flow. The axis system and flow orientation are identical to those in Fig. 6; the orientation shown corresponds with $\phi=3 \pi / 2$.

where the scaled period $\dot{\gamma} \tau$ and the equivalent axis ratio $\rho$ are functions of the relative dimensions of the cap. In the simulations, a hemi-spherical cap, $\Theta=\pi / 2$, is modelled by primary particles distributed over the surface by the DistMesh routine. The minimum distance between any two particles is again used to define their diameter. Whereas an infinitely thin shell was assumed by Dorrepaal, the simulated shell will only converge to this limit when the cap's radius far exceeds the particle's diameter, i.e., for $N \rightarrow \infty$. The procedure of Sec. II A is used to determine the mobility matrix that enters in the actual simulation of the motion, supplemented by a shift of the reference point to the hydrodynamic center of the cap by the procedure of Appendix G. The simulated periods of bodies of 526 and 2051 primary particles closely approximate the analytical periods, being shorter by $0.4 \%$ and $0.03 \%$, respectively.

Due to the lack of fore-aft symmetry, resulting in a nonzero $\boldsymbol{\mu}_{\mathcal{E}}^{v}$, the hydrodynamic center moves periodically. ${ }^{50}$ The paths traced by three points on the symmetry axis of a simulated hemi-spherical cap are shown in Fig. 12. Unlike for points on the ellipsoidal body at $\theta=\pi / 2$, see Fig. 6 , the paths are non-circular and there is no stationary point on the body. For a quantitative comparison with theory, Dorrepaal's point $Q$ on the symmetry axis (the red bead in Fig. 12) is selected. The simulated motions of this point, for two caps with differing numbers of primary particles, agree well with theory, as shown in Fig. 13, indicating once more that the rotational and translational motions are simulated correctly. In the absence of Brownian motion, Eq. (19) can be applied using any point in the body frame as the reference point. Tests with randomly chosen reference points indeed recovered the orbits depicted in Figs. 12 and 13 (data not shown).

Evaluation of the Einstein coefficient, based on the volume $V_{\mathrm{c}}=\frac{2}{3} \pi R^{3}$ enclosed by the cap, yields a periodically undulating $B_{x y}$ akin to that for the ellipsoid (data not shown). The main differences are a considerable reduction and widening of the symmetric double peaks flanking the minima at $\phi= \pm \pi / 2$. These minima are again approximately equally low as the minima attained in the flow-aligned state, $\phi=0$ and $\phi=\pi$, and all minima undershoot the constant value of 2.5 attained for a sphere. 


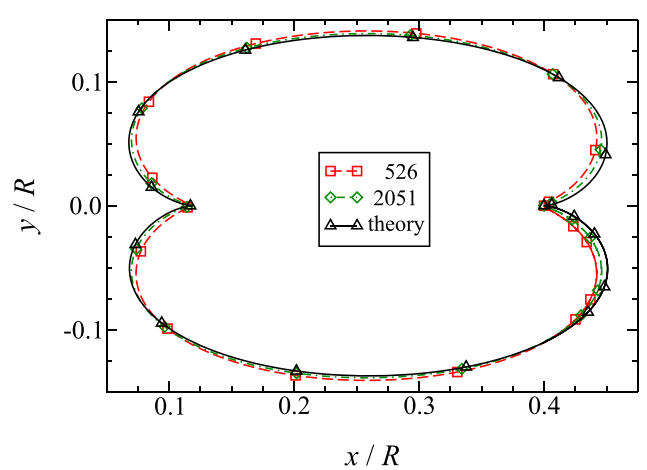

FIG. 13. Orbit of the center of free rotation of a hemi-spherical cap in a shear flow, showing simulations with two differing numbers of primary particles and Dorrepaal's theory. The markers are equidistant in time and ideally should have coalesced. The center of the cap (the starting point of the arrow in Fig. 12) coincides with the origin of the coordinate system for $\phi=0$.

\section{SUMMARY AND CONCLUSIONS}

The Brownian motion of a rigid arbitrary shaped colloidal body is conveniently simulated using Cartesian coordinates for the position of the hydrodynamic center and unit quaternions for the orientation of the body. As shown by Ilie et al. ${ }^{42}$ the use of quaternions - in combination with a unit-length constraint-simplifies the Brownian equation of motion in the Itô representation by eliminating several terms. Whereas Ilie et al. assumed a $(6 \times 6)$ mobility matrix consisting of two $(3$ $\times 3$ ) blocks for the translational and rotational motion, respectively, the formalism is expanded here to an equation of motion based on a $(11 \times 11)$ grand mobility matrix; see Eq. (19). The advantages of this expansion are the proper inclusion of translation-rotation coupling beyond the rotation-dependence of the translational mobility, the ability to simulate bodies in linear flow fields and access to the body-induced stress in the fluid. The grand mobility matrix is constructed by representing (the surface of) the body by a collection of spherical primary particles, followed by a weighted summation of the hydrodynamic interactions over all combinations of two primary particles. A code to calculate the grand mobility matrix is available at www2.msm.ctw.utwente.nl/Oseen11. Simulation results employing this approach to differing particles of various complexities yield excellent agreement with theory, recovering the Boltzmann distribution and Favro's rotational relaxation ${ }^{44}$ for colloids in quiescent fluids and the periodic orbits derived by Jeffery ${ }^{3}$ and Dorrepaal ${ }^{43}$ for ellipsoids and spherical caps, respectively, immersed in shear flows in the absence of thermal noise. The proposed framework enables computational studies on the complex dynamics of bodies under the combined effects of potential forces, flow, and Brownian motion, for which only approximate theoretical descriptions exist to date, like the Einstein viscosity of dilute suspensions of non-spherical colloids. ${ }^{10,49,51}$

\section{ACKNOWLEDGMENTS}

We thank Professor Stefan Luding for stimulating discussions. This work is part of the "Computational sciences for energy research" of the Netherlands Organisation for Scientific Research (NWO) under Project No. 13CSER060. This research programme is co-financed by Shell Global Solutions International B.V.

\section{APPENDIX A: PAIR MOBILITIES}

The generalized mobility and resistance tensors appearing in the various equations of the main text are of mixed units. With $a$ being the radius of the particle, $\tau$ being the unit of time, and $\eta_{\mathrm{s}}$ being the solvent viscosity, the pair mobility problem can be re-expressed as

$$
\left(\begin{array}{c}
\left(\mathbf{v}_{i}-\mathbf{v}_{i}^{\infty}\right) \tau / a \\
\left(\omega_{i}-\omega^{\infty}\right) \tau \\
-\mathbf{E}^{\infty} \tau
\end{array}\right)=\frac{1}{6 \pi} \sum_{j=1}^{N} \boldsymbol{\mu}_{j}^{i}\left(\begin{array}{c}
\mathbf{f}_{j} \tau /\left(\eta_{\mathrm{s}} a^{2}\right) \\
\boldsymbol{\tau}_{j} \tau /\left(\eta_{\mathrm{s}} a^{3}\right) \\
\mathbf{S}_{j} \tau /\left(\eta_{\mathrm{s}} a^{3}\right)
\end{array}\right) .
$$

All elements of the generalized vectors (between large brackets) on the l.h.s. and r.h.s. of this expression are dimensionless, and hence all elements of the tensors $\boldsymbol{\mu}_{j}^{i}$ are also dimensionless. Following the steps outlined in the main text, the dimensionless mobility problem of the cluster becomes

$$
\left(\begin{array}{c}
\left(\mathbf{v}-\mathbf{v}^{\infty(s)}\right) \tau / a \\
\left(\omega-\omega^{\infty}\right) \tau \\
\mathcal{S} \tau /\left(\eta_{\mathrm{s}} a^{3}\right)
\end{array}\right)=\frac{1}{6 \pi} \mu\left(\begin{array}{c}
\mathbf{f} \tau /\left(\eta_{\mathrm{s}} a^{2}\right) \\
\tau \tau /\left(\eta_{\mathrm{s}} a^{3}\right) \\
-\mathcal{E}^{\infty} \tau
\end{array}\right) .
$$

The corresponding dimensionless equation of motion is readily obtained.

The tensorial character of the pair mobility matrix $\boldsymbol{\mu}_{j}^{i}$ imposes the structure of the hydrodynamic interactions between particles $i$ and $j$, with difference vector $\mathbf{r}^{i j}=\mathbf{x}_{j}-\mathbf{x}_{i}$, parallel unit vector $\hat{\mathbf{r}}^{i j}=\mathbf{r}^{i j} / r_{i j}$, traceless dyadic $\mathbf{d}^{i j}=\hat{\mathbf{r}}^{i j} \otimes$ $\hat{\mathbf{r}}^{i j}-\frac{1}{3} \mathbf{1}$, and perpendicular projection $\mathbf{p}^{i j}=\mathbf{1}-\hat{\mathbf{r}}^{i j} \otimes \hat{\mathbf{r}}^{i j}$, where for compactness of notation the particle-pair label is denoted as superscript to the vectors and matrices. Using auxiliary functions $x, y$, and $z$ in the dimensionless distance $\tilde{r}_{i j}=r_{i j} / a$, the elements of the mobility matrix read as $^{5,24,52,53}$

$$
\begin{aligned}
\mu_{f, j, \beta}^{v, i, \alpha}= & x_{f, j}^{v, i} \hat{r}_{\alpha}^{i j} \hat{r}_{\beta}^{i j}+y_{f, j}^{v, i} p_{\alpha \beta}^{i j}, \\
\mu_{f, j, \beta}^{\omega, i, \alpha}= & y_{f, j}^{\omega, i} \epsilon_{\alpha \beta \gamma} \hat{r}_{\gamma}^{i j}, \\
\mu_{\tau, j, \beta}^{\omega, i, \alpha}= & x_{\tau, j}^{\omega, i} \hat{r}_{\alpha}^{i j} \hat{r}_{\beta}^{i j}+y_{\tau, j}^{\omega, i} p_{\alpha \beta}^{i j}, \\
\mu_{f, j, \gamma}^{E, i, \alpha \beta}= & x_{f, j}^{E, i} d_{\alpha \beta}^{i j} \hat{r}_{\gamma}^{i j}+y_{f, j}^{E, i}\left(\hat{r}_{\alpha}^{i j} p_{\beta \gamma}^{i j}+\hat{r}_{\beta}^{i j} p_{\alpha \gamma}^{i j}\right), \\
\mu_{\tau, j, \gamma}^{E, i, \alpha \beta}= & y_{\tau, j}^{E, i}\left(\hat{r}_{\alpha}^{i j} \epsilon_{\beta \gamma \delta}+\hat{r}_{\beta}^{i j} \epsilon_{\alpha \gamma \delta}\right) \hat{r}_{\delta}^{i j}, \\
\mu_{S, j, \gamma \delta}^{E, i, \alpha \beta}= & x_{S, j}^{E, i} d_{\alpha \beta}^{i j} d_{\gamma \delta}^{i j}+y_{S, j}^{E, i}\left[\hat{r}_{\alpha}^{i j} p_{\beta \gamma}^{i j} \hat{r}_{\delta}^{i j}+\hat{r}_{\alpha}^{i j} p_{\beta \delta}^{i j} \hat{r}_{\gamma}^{i j}+\hat{r}_{\beta}^{i j} p_{\alpha \gamma}^{i j} \hat{r}_{\delta}^{i j}\right. \\
& \left.+\hat{r}_{\beta}^{i j} p_{\alpha \delta}^{i j} \hat{r}_{\gamma}^{i j}\right]+z_{S, j}^{E, i}\left[p_{\alpha \gamma}^{i j} p_{\beta \delta}^{i j}+p_{\beta \gamma}^{i j} p_{\alpha \delta}^{i j}-p_{\alpha \beta}^{i j} p_{\gamma \delta}^{i j}\right]
\end{aligned}
$$

with the remaining elements following from the symmetry relations

$$
\begin{aligned}
\mu_{\tau, i, \alpha}^{v, j, \beta} & =\mu_{f, j, \beta}^{\omega, i, \alpha}, \\
\mu_{S, i, \alpha \beta}^{v, j, \gamma} & =\mu_{f, j, \gamma}^{E, i, \alpha \beta}, \\
\mu_{S, i, \alpha \beta}^{\omega, j, \gamma} & =\mu_{\tau, j, \gamma}^{E, i, \alpha \beta} .
\end{aligned}
$$


In the Rotne-Prager-Yamakawa approximation, the auxiliary functions are given by

$$
\begin{aligned}
x_{f, j}^{v, i} & =\delta_{i j}+\left(1-\delta_{i j}\right)\left(\frac{3}{2} \tilde{r}_{i j}^{-1}-\tilde{r}_{i j}^{-3}\right), \\
y_{f, j}^{v, i} & =\delta_{i j}+\left(1-\delta_{i j}\right)\left(\frac{3}{4} \tilde{r}_{i j}^{-1}+\frac{1}{2} \tilde{r}_{i j}^{-3}\right), \\
y_{f, j}^{\omega, i} & =-\left(1-\delta_{i j}\right) \frac{3}{4} \tilde{r}_{i j}^{-2}, \\
x_{\tau, j}^{\omega, i} & =\frac{3}{4} \delta_{i j}+\left(1-\delta_{i j}\right) \frac{3}{4} \tilde{r}_{i j}^{-3}, \\
y_{\tau, j}^{\omega, i} & =\frac{3}{4} \delta_{i j}-\left(1-\delta_{i j}\right) \frac{3}{8} \tilde{r}_{i j}^{-3}, \\
x_{f, j}^{E, i} & =\left(1-\delta_{i j}\right)\left(\frac{9}{4} \tilde{r}_{i j}^{-2}-\frac{18}{5} \tilde{r}_{i j}^{-4}\right), \\
y_{f, j}^{E, i} & =\left(1-\delta_{i j}\right) \frac{6}{5} \tilde{r}_{i j}^{-4}, \\
y_{\tau, j}^{E, i} & =-\left(1-\delta_{i j}\right) \frac{9}{8} \tilde{r}_{i j}^{-3}, \\
x_{S, j}^{E, i} & =\frac{27}{20} \delta_{i j}-\left(1-\delta_{i j}\right)\left(\frac{27}{4} \tilde{r}_{i j}^{-3}-\frac{81}{5} \tilde{r}_{i j}^{-5}\right), \\
y_{S, j}^{E, i} & =\frac{9}{20} \delta_{i j}+\left(1-\delta_{i j}\right)\left(\frac{9}{8} \tilde{r}_{i j}^{-3}-\frac{18}{5} \tilde{r}_{i j}^{-5}\right), \\
z_{S, j}^{E, i} & =\frac{9}{20} \delta_{i j}+\left(1-\delta_{i j}\right) \frac{9}{10} \tilde{r}_{i j}^{-5} .
\end{aligned}
$$

One may readily substitute these functions with higher-order approximations. $^{5}$

\section{APPENDIX B: THE BASIS MATRICES}

Like the strain rate in Eq. (3), the stress is converted between matrix and vector representations by

$$
\begin{aligned}
\mathbf{S} & =\mathbf{e}_{\kappa}^{S} \mathcal{S}_{K}, \quad S_{\alpha \beta}=\left(\mathbf{e}_{\kappa}^{S}\right)_{\alpha \beta} \mathcal{S}_{K}, \\
\mathcal{S}_{K} & =\mathbf{e}_{S}^{\kappa}: \mathbf{S}, \quad \mathcal{S}_{K}=\left(\mathbf{e}_{S}^{\kappa}\right)_{\alpha \beta} S_{\alpha \beta},
\end{aligned}
$$

where the Einstein summation convention is used, with Greek indices from the start of the alphabet $(\alpha, \beta, \ldots)$ running over the three Cartesian directions and Greek indices from the middle of the alphabet $(\kappa, \lambda)$ running over 1 through 5 . For the five components defining the symmetric traceless stress tensor, it proves convenient to select the three shear-stresses, $\mathcal{S}_{1}=S_{x y}$, $\mathcal{S}_{2}=S_{x z}$, and $\mathcal{S}_{3}=S_{y z}$, in combination with the first and second normal stress differences, $\mathcal{S}_{4}=S_{x x}-S_{y y}$ and $\mathcal{S}_{5}=S_{y y}-S_{z z}$, respectively. The corresponding five basis matrices (akin to basis vectors) to convert the stress from vector $\mathcal{S}$ to matrix $\mathbf{S}$ then read as

$$
\begin{array}{ll}
\mathbf{e}_{1}^{S}=\left(\begin{array}{lll}
0 & 1 & 0 \\
1 & 0 & 0 \\
0 & 0 & 0
\end{array}\right), & \mathbf{e}_{2}^{S}=\left(\begin{array}{lll}
0 & 0 & 1 \\
0 & 0 & 0 \\
1 & 0 & 0
\end{array}\right), \\
\mathbf{e}_{3}^{S}=\left(\begin{array}{lll}
0 & 0 & 0 \\
0 & 0 & 1 \\
0 & 1 & 0
\end{array}\right), & \mathbf{e}_{4}^{S}=\frac{1}{3}\left(\begin{array}{rrr}
2 & 0 & 0 \\
0 & -1 & 0 \\
0 & 0 & -1
\end{array}\right), \\
\mathbf{e}_{5}^{S}=\frac{1}{3}\left(\begin{array}{ccc}
1 & 0 & 0 \\
0 & 1 & 0 \\
0 & 0 & -2
\end{array}\right) . &
\end{array}
$$

Since these basis matrices are not orthogonal, in the sense that $\mathbf{e}_{\kappa}^{S}: \mathbf{e}_{\lambda}^{S} \neq \delta_{\kappa \lambda}$ with $\delta$ being the Kronecker delta, the conversion of the stress from matrix $\mathbf{S}$ to vector $\mathcal{S}$ requires the reciprocal basis

$$
\begin{aligned}
& \mathbf{e}_{S}^{1}=\frac{1}{2} \mathbf{e}_{1}^{S}, \quad \mathbf{e}_{S}^{2}=\frac{1}{2} \mathbf{e}_{2}^{S}, \quad \mathbf{e}_{S}^{3}=\frac{1}{2} \mathbf{e}_{3}^{S}, \\
& \mathbf{e}_{S}^{4}=\left(\begin{array}{ccc}
1 & 0 & 0 \\
0 & -1 & 0 \\
0 & 0 & 0
\end{array}\right), \quad \mathbf{e}_{S}^{5}=\left(\begin{array}{ccc}
0 & 0 & 0 \\
0 & 1 & 0 \\
0 & 0 & -1
\end{array}\right),
\end{aligned}
$$

as is readily verified using Eq. (B1).

The particle-particle grand mobility matrices in Eq. (1) satisfy a number of symmetry rules, derivable from the Lorentz reciprocal theorem. ${ }^{5}$ Consequently, when choosing the (dual) basis matrices for the strain rate as

$$
\begin{aligned}
\mathbf{e}_{\kappa}^{E} & =\mathbf{e}_{S}^{K}, \\
\mathbf{e}_{E}^{K} & =\mathbf{e}_{\kappa}^{S},
\end{aligned}
$$

the particle-particle grand mobility matrices in Eq. (4) will inherit these symmetries, and the cluster's grand mobility matrix in Eq. (10) will be symmetric. The five elements of the strain rate vector then represent the three shear rates, $\mathcal{E}_{1}^{\infty}=2 E_{x y}^{\infty}=\partial v_{x}^{\infty} / \partial y+\partial v_{y}^{\infty} / \partial x, \mathcal{E}_{2}^{\infty}=2 E_{x z}^{\infty}$, and $\mathcal{E}_{3}^{\infty}=2 E_{y z}^{\infty}$, as well as the two extensional rates $\mathcal{E}_{4}^{\infty}=E_{x x}^{\infty}=\partial v_{x}^{\infty} / \partial x$ and $\mathcal{E}_{5}^{\infty}=-E_{z z}^{\infty}$, respectively. The imposition of symmetry is convenient, but not compulsory, to the approach taken in this paper.

\section{APPENDIX C: GRAND RESISTANCE MATRIX}

In the vectorial representation of the strain rate, the background flow field experienced by particle $j$ can be expressed as a sum of matrix-vector products through

$$
\mathbf{v}^{\infty}\left(\mathbf{x}_{j}\right)=\mathbf{v}^{\infty}(\mathbf{x})+\varphi_{j} \mathcal{E}^{\infty}-\varepsilon_{j} \omega^{\infty},
$$

which is made of the $(3 \times 5)$ matrices

$$
\varphi_{j, \alpha \kappa}=\varphi_{\alpha \kappa}\left(\mathbf{r}_{j}\right)=\sum_{\beta}\left(\mathbf{e}_{\kappa}^{E}\right)_{\alpha \beta} r_{j, \beta}
$$

and the $(3 \times 3)$ matrices

$$
\varepsilon_{j, \alpha \beta}=\varepsilon_{\alpha \beta}\left(\mathbf{r}_{j}\right)=\epsilon_{\alpha \gamma \beta} r_{j, \gamma},
$$

with $\epsilon$ being the Levi-Civita tensor. Inserting these velocities in Eq. (6) yields minus the hydrodynamic forces on the particles, which are readily summed, see Eq. (8a), to obtain minus the total hydrodynamic force on the cluster. The force-related submatrices in the grand mobility of the cluster are then extracted as

$$
\begin{aligned}
\boldsymbol{\xi}_{v}^{f} & =\sum_{i, j=1}^{N} \xi_{v, j}^{f, i}, \\
\boldsymbol{\xi}_{\omega}^{f} & =\sum_{i, j=1}^{N}\left[\xi_{\omega, j}^{f, i}-\boldsymbol{\xi}_{v, j}^{f, i} \boldsymbol{\varepsilon}_{j}\right], \\
\boldsymbol{\xi}_{\mathcal{E}}^{f} & =\sum_{i, j=1}^{N}\left[\xi_{\mathcal{E}, j}^{f, i}+\xi_{v, j}^{f, i} \boldsymbol{\varphi}_{j}\right] .
\end{aligned}
$$


Similarly, insertion of the hydrodynamic forces into the summation expression for the torques, see Eq. (8b), yields

$$
\begin{aligned}
\boldsymbol{\xi}_{v}^{\tau} & =\sum_{i, j=1}^{N}\left[\boldsymbol{\xi}_{v, j}^{\tau, i}+\boldsymbol{\varepsilon}_{i} \boldsymbol{\xi}_{v, j}^{f, i}\right] \\
\boldsymbol{\xi}_{\omega}^{\tau} & =\sum_{i, j=1}^{N}\left[\boldsymbol{\xi}_{\omega, j}^{\tau, i}-\boldsymbol{\xi}_{v, j}^{\tau, i} \boldsymbol{\varepsilon}_{j}-\boldsymbol{\varepsilon}_{i} \boldsymbol{\xi}_{v, j}^{f, i} \boldsymbol{\varepsilon}_{j}+\boldsymbol{\varepsilon}_{i} \boldsymbol{\xi}_{\omega, j}^{f, i}\right] \\
\boldsymbol{\xi}_{\mathcal{E}}^{\tau} & =\sum_{i, j=1}^{N}\left[\boldsymbol{\xi}_{\mathcal{E}, j}^{\tau, i}+\boldsymbol{\xi}_{v, j}^{\tau, i} \boldsymbol{\varphi}_{j}+\boldsymbol{\varepsilon}_{i} \boldsymbol{\xi}_{v, j}^{f, i} \boldsymbol{\varphi}_{j}+\boldsymbol{\varepsilon}_{i} \boldsymbol{\xi}_{\mathcal{E}, j}^{f, i}\right]
\end{aligned}
$$

In the vectorial representation of the stress, the addition rule of Eq. (8c) can be expressed as a sum of matrix-vector products through

$$
\mathcal{S}=\sum_{i=1}^{N}\left[\mathcal{S}_{i}+\boldsymbol{\psi}_{i} \mathbf{f}_{i}\right],
$$

with the $(5 \times 3)$ matrices

$$
\psi_{i, \kappa \alpha}=\psi_{\kappa \alpha}\left(\mathbf{r}_{i}\right)=\sum_{\beta} r_{i, \beta}\left(\mathbf{e}_{S}^{\kappa}\right)_{\beta \alpha} .
$$

Then

$$
\begin{aligned}
& \boldsymbol{\xi}_{v}^{\mathcal{S}}=\sum_{i, j=1}^{N}\left[\boldsymbol{\xi}_{v, j}^{\mathcal{S}, i}+\boldsymbol{\psi}_{i} \boldsymbol{\xi}_{v, j}^{f, i}\right], \\
& \boldsymbol{\xi}_{\omega}^{\mathcal{S}}=\sum_{i, j=1}^{N}\left[\boldsymbol{\xi}_{\omega, j}^{\mathcal{S}, i}-\boldsymbol{\xi}_{v, j}^{\mathcal{S}, i} \boldsymbol{\varphi}_{j}-\boldsymbol{\psi}_{i} \boldsymbol{\xi}_{v, j}^{f, i}+\boldsymbol{\psi}_{i} \boldsymbol{\xi}_{\omega, j}^{f, i}\right] \\
& \boldsymbol{\xi}_{\mathcal{E}}^{\mathcal{S}}=\sum_{i, j=1}^{N}\left[\boldsymbol{\xi}_{\mathcal{E}, j}^{\mathcal{S}, i}+\boldsymbol{\xi}_{v, j}^{\mathcal{S}, i} \boldsymbol{\varphi}_{j}+\boldsymbol{\psi}_{i} \boldsymbol{\xi}_{v, j}^{f, i} \boldsymbol{\varphi}_{j}+\boldsymbol{\psi}_{i} \boldsymbol{\xi}_{\mathcal{E}, j}^{f, i}\right] .
\end{aligned}
$$

From the symmetry of the $(6 \times 6)$ top-left sub-matrix of the particle-based grand resistance matrices, it readily follows that the $(6 \times 6)$ top-left sub-matrix of the cluster's grand resistance matrix is also symmetric. Retainment of the symmetries of the particle-based sub-matrices related to the stress and strain, however, is subject to the chosen basis matrices; see Appendix B.

\section{APPENDIX D: PARTIAL INVERSION}

To solve $\mathbf{B}$ and $\mathbf{C}$ in the relation

$$
\left(\begin{array}{l}
\mathbf{A} \\
\mathbf{B}
\end{array}\right)=\left(\begin{array}{ll}
\mathbf{Q} & \mathbf{R} \\
\mathbf{S} & \mathbf{T}
\end{array}\right)\left(\begin{array}{l}
\mathbf{C} \\
\mathbf{D}
\end{array}\right),
$$

one first solves $\mathbf{C}$ from the top line, followed by the substitution of this result in the bottom line, yielding

$$
\left(\begin{array}{l}
\mathbf{C} \\
\mathbf{B}
\end{array}\right)=\left(\begin{array}{cc}
\mathbf{Q}^{-1} & -\mathbf{Q}^{-1} \mathbf{R} \\
\mathbf{S Q}^{-1} & \mathbf{T}-\mathbf{S Q}^{-1} \mathbf{R}
\end{array}\right)\left(\begin{array}{l}
\mathbf{A} \\
\mathbf{D}
\end{array}\right) .
$$

In the context of Eq. (9), $\mathbf{B}$ and $\mathbf{C}$ refer to the five hydrodynamic stresses and the six generalized velocities of the cluster, respectively, while $\mathbf{A}$ and $\mathbf{D}$ represent the six generalized conservative forces on the cluster and minus the strain rate of the fluid, respectively.

\section{APPENDIX E: QUATERNIONS}

A rotation matrix in three-dimensional space can be expressed in terms of the unit quaternion four-vector, $\mathbf{q}=\left(q_{0}\right.$, $\left.q_{1}, q_{2}, q_{3}\right)$, with $|\mathbf{q}|=1$, where for the conversion from the body frame (b) to the space frame (s) we use

$$
\mathbf{A}_{(\mathrm{b})}^{(\mathrm{s})}=\left(\begin{array}{ccc}
q_{0}^{2}+q_{1}^{2}-q_{2}^{2}-q_{3}^{2} & 2 q_{1} q_{2}-2 q_{0} q_{3} & 2 q_{1} q_{3}+2 q_{0} q_{2} \\
2 q_{1} q_{2}+2 q_{0} q_{3} & q_{0}^{2}-q_{1}^{2}+q_{2}^{2}-q_{3}^{2} & 2 q_{2} q_{3}-2 q_{0} q_{1} \\
2 q_{1} q_{3}-2 q_{0} q_{2} & 2 q_{2} q_{3}+2 q_{0} q_{1} & q_{0}^{2}-q_{1}^{2}-q_{2}^{2}+q_{3}^{2}
\end{array}\right) .
$$

The conversion from the space frame to the body frame is realised by $\mathbf{A}_{(\mathrm{s})}^{(\mathrm{b})}$, which is simply the transposed (as well as the inverse) of the above matrix. In the simulation algorithm, the conversion of angular velocities in the space frame to quaternion velocities is realized by

$$
\mathbf{B}_{(\mathrm{s})}^{\dot{q}}=\frac{\partial \dot{\mathbf{q}}}{\partial \omega^{(\mathrm{s})}}=\frac{1}{2 q^{4}}\left(\begin{array}{rrr}
-q_{1} & -q_{2} & -q_{3} \\
q_{0} & q_{3} & -q_{2} \\
-q_{3} & q_{0} & q_{1} \\
q_{2} & -q_{1} & q_{0}
\end{array}\right),
$$

with $q=|\mathbf{q}|$, and the conversion of angular velocities in the body frame to quaternion velocities is realized by

$$
\mathbf{B}_{(\mathrm{b})}^{\dot{q}}=\frac{\partial \dot{\mathbf{q}}}{\partial \boldsymbol{\omega}^{(\mathrm{b})}}=\frac{1}{2 q^{4}}\left(\begin{array}{rrr}
-q_{1} & -q_{2} & -q_{3} \\
q_{0} & -q_{3} & q_{2} \\
q_{3} & q_{0} & -q_{1} \\
-q_{2} & q_{1} & q_{0}
\end{array}\right) .
$$

One readily shows that the latter two matrices are related by $\mathbf{B}_{(\mathrm{b})}^{\dot{q}}=\mathbf{B}_{(\mathrm{s})}^{\dot{q}} \mathbf{A}_{(\mathrm{b})}^{(\mathrm{s})}$.

\section{APPENDIX F: BROWNIAN EQUATION OF MOTION}

In deriving the rigid-body equation of motion from the generic Brownian equation of motion, see Eqs. (12) and (19), we start by noting that the intended set of coordinates, 
$\mathbf{Q}=\left(\mathbf{x}^{\mathrm{T}}, \mathbf{q}^{\mathrm{T}}\right)^{\mathrm{T}}$, includes one surplus coordinate relative to the six coordinates needed to describe rigid body translation and rotation. One readily sees from Eq. (E1) that the four quaternions describe rotation as well as enlargement, $\mathbf{A}_{(\mathbf{b})}^{(\mathrm{s})}(\mathbf{q})=q^{2} \mathbf{A}_{(\mathbf{b})}^{(\mathrm{s})}(\mathbf{q} / q)$. For pure rotations, the quaternion vector must be constrained to unit length, $q=1$, throughout the simulation. Furthermore, as noted by Ilie et al. ${ }^{42}$ a Brownian equation of motion using quaternions requires a mobility matrix that decouples enlargement from translation and rotation. Assuming a quiescent solvent for convenience, we construct the mobility matrix entering the generic expression as

$$
\boldsymbol{\mu}_{Q}=\left(\begin{array}{cc}
\mathbf{A}_{(\mathrm{b})}^{(\mathrm{s})} & \mathbf{0} \\
\mathbf{0} & \mathbf{B}_{(\mathrm{b})}^{\dot{q}}
\end{array}\right)\left(\begin{array}{cc}
\boldsymbol{\mu}_{f}^{v} & \boldsymbol{\mu}_{\tau}^{v} \\
\boldsymbol{\mu}_{f}^{\omega} & \boldsymbol{\mu}_{\tau}^{\omega}
\end{array}\right)_{(\mathrm{b})}^{(\mathrm{b})}\left(\begin{array}{cc}
\mathbf{A}_{(\mathrm{b})}^{(\mathrm{s})} & \mathbf{0} \\
\mathbf{0} & \mathbf{B}_{(\mathrm{b})}^{\dot{q}}
\end{array}\right)^{\mathrm{T}}
$$

where the central matrix on the r.h.s. is the Cartesian bodybased mobility matrix, the matrix to its left converts bodybased velocities to space-based and quaternion velocities, and the transposed matrix to its right is dictated by the symmetry of $\boldsymbol{\mu}_{Q}$. Since the columns of $\mathbf{B}_{(\mathrm{b})}^{\dot{q}}$ are orthogonal to $\mathbf{q}$, see Eq. (E3), the left-most matrix in the triple product ensures that this mobility matrix conserves the length of the quaternion vector (in the limit of $\Delta t \rightarrow 0$ ). The restrictions on the use of quaternions are therefore met.

Upon insertion of the above mobility matrix in Eq. (12), it readily follows from $\left(\mathbf{A}_{(\mathrm{b})}^{(\mathrm{s})}\right)^{\mathrm{T}}=\mathbf{A}_{(\mathrm{s})}^{(\mathrm{b})}$ that conservative forces in the space frame, $f_{\alpha}^{(\mathrm{s})}=-\partial \Phi / \partial Q_{\alpha}=-\partial \Phi / \partial x_{\alpha}$ with $\alpha \in\{1,2,3\}$, are converted to forces in the body frame before left-multiplication with the body-based mobility matrix. The generalized forces with respect to the four rotational coordinates, $f_{\rho+4}^{q}=-\partial \Phi / \partial Q_{\rho+4}=-\partial \Phi / \partial q_{\rho}$ with $\rho \in\{0,1,2,3\}$, are converted by $\left(\mathbf{B}_{(\mathbf{b})}^{\dot{q}}\right)^{\mathrm{T}}$ before left-multiplication with the body-based mobility matrix. Using the matrices introduced in Appendix E, it follows that

$$
\begin{aligned}
\left(\mathbf{B}_{(\mathrm{b})}^{\dot{q}}\right)^{\mathrm{T}} \frac{\partial \Phi}{\partial \mathbf{q}} & =\left(\mathbf{A}_{(\mathrm{b})}^{(\mathrm{s})}\right)^{\mathrm{T}}\left(\mathbf{B}_{(\mathrm{s})}^{\dot{q}}\right)^{\mathrm{T}} \frac{\partial \Phi}{\partial \mathbf{q}} \\
& =\mathbf{A}_{(\mathrm{s})}^{(\mathrm{b})} \frac{\partial \Phi}{\partial \mathbf{q}} \frac{\partial \dot{\mathbf{q}}}{\partial \omega^{(\mathrm{s})}}=\mathbf{A}_{(\mathrm{s})}^{(\mathrm{b})} \frac{\partial \Phi}{\partial \psi^{(\mathrm{s})}}
\end{aligned}
$$

where the rotation vector $\psi^{(\mathrm{s})}$ collects the rotation angles around the three space-based coordinate axes, with rates of change $\dot{\psi}^{(\mathrm{s})}=\omega^{(\mathrm{s})}$. The final derivative of the potential energy with respect to $\psi^{(\mathrm{s})}$ yields minus the usual torque vector in the space frame, $\tau^{(\mathrm{s})}$. Hence, the above derivation shows that the conservative potential enters Eqs. (12) and (19) in the same way, with the latter form being easier to calculate. Note that the rows of $\left(\mathbf{B}_{(\mathrm{b})}^{\dot{q}}\right)^{\mathrm{T}}$ are perpendicular to $\mathbf{q}$; hence, conservative forces that strive to enlarge the body, i.e., force components $\partial \Phi / \partial \mathbf{q}$ parallel to $\mathbf{q}$, are eliminated by the third matrix on the r.h.s. to Eq. (F1).

Using the above mobility matrix $\boldsymbol{\mu}_{Q}$, we next derive the divergence term in Eq. (12). The derivative of $\mu_{Q}$ with respect to the position $\mathbf{x}$ is clearly zero; hence, neither $\boldsymbol{\mu}_{f}^{v}$ nor $\boldsymbol{\mu}_{f}^{\omega}$ contributes to the divergence. A straightforward but laborious derivation, using the matrices of Appendix E and the symmetry of $\boldsymbol{\mu}_{\tau(\mathrm{b})}^{\omega(\mathrm{b})}$, yields the vector

$$
\frac{\partial\left(\boldsymbol{\mu}_{Q}\right)_{\sigma \phi}}{\partial Q_{\phi}}=\left(\begin{array}{c}
-\left(\mathbf{A}_{(\mathrm{b})}^{(\mathrm{s})}\right)_{\sigma \alpha} \epsilon_{\alpha \beta \gamma}\left(\boldsymbol{\mu}_{\tau(\mathrm{b})}^{v(\mathrm{~b})}\right)_{\beta \gamma} / q^{4} \\
q_{\sigma-4} \operatorname{Tr}\left(\boldsymbol{\mu}_{\tau(\mathrm{b})}^{\omega(\mathrm{b})}\right) /\left(4 q^{8}\right)
\end{array}\right),
$$

where the top line applies to translational coordinates, i.e., $\sigma \in\{1,2,3\}$, while the bottom line applies to rotational coordinates, i.e., $\sigma \in\{4,5,6,7\}$. Furthermore, $\{\alpha, \beta, \gamma\}$ $\in\{1,2,3\}, \phi \in\{1, \ldots, 7\}$, and $\operatorname{Tr}$ denotes the trace. The first three elements of this vector vanish identically when the matrix $\boldsymbol{\mu}_{\tau(\mathrm{b})}^{v(\mathrm{~b})}$ is symmetric, as is the case when the mobility matrix is evaluated relative to the hydrodynamic center of the cluster; see Appendix G. This selection of the body-based origin is assumed in Eq. (19). The last four elements of the vector contribute a displacement parallel to $\mathbf{q}$ to the rotational coordinates. Since this displacement is parallel to the constraint term $\lambda \mathbf{q}$ maintaining the unit length of $\mathbf{q}$, it will be eliminated by the constraint - thereby removing the need to determine the contribution of the divergence term to the rotational motion.

The metric $g_{Q}$ or Jacobian $J_{Q}=g_{Q}^{1 / 2}$ is used to measure the (squared) volume of elements $d \mathbf{Q}$ in a general coordinate space. To determine the metric when using Cartesian coordinates $\mathbf{x}$ and quaternions $\mathbf{q}$ to describe a rigid body, we switch to the proportional mass-metric $M_{Q}=\left|\mathbf{M}_{Q}\right|$. The latter tensor relates the kinetic energy $K$ to the generalized velocities, which for a rigid cluster with body-based density distribution $\rho^{(\mathrm{b})}(\mathbf{r})$ reads as

$$
K=\frac{1}{2} \dot{\mathbf{Q}}^{\mathrm{T}} \mathbf{M}_{Q} \dot{\mathbf{Q}}=\frac{1}{2} \int\left[\dot{\mathbf{x}}+\frac{\partial \mathbf{A}_{(\mathrm{b})}^{(\mathrm{s})}}{\partial \mathbf{q}} \dot{\mathbf{q}} \mathbf{r}\right]^{2} \rho^{(\mathrm{b})}(\mathbf{r}) d \mathbf{r} .
$$

In the creeping flow limit, the mass distribution is irrelevant for the motion of the cluster. We therefore select a density such that the center of mass coincides with the origin of the body frame, the three eigenvectors of the inertia tensor are parallel to the axes of the body frame, and the three corresponding eigenvalues are identical. This renders the mass-metric block diagonal, combining a constant translational block with an orientation-dependent rotational block,

$$
\left(\mathbf{M}_{Q}\right)_{\rho \sigma} \propto \frac{\partial\left(\mathbf{A}_{(\mathrm{b})}^{(\mathrm{s})}\right)_{\alpha \beta}}{\partial q_{\rho-4}} \frac{\partial\left(\mathbf{A}_{(\mathrm{b})}^{(\mathrm{s})}\right)_{\alpha \beta}}{\partial q_{\sigma-4}},
$$

with $\{\rho, \sigma\} \in\{4,5,6,7\}$. After some work follows

$$
g_{Q} \propto M_{Q} \propto|\mathbf{q}|^{8}
$$

The resulting derivative of the metric entering the equation of motion, see Eqs. (12) and (13), is parallel to $\mathbf{q}$ and therefore is eliminated by the $\left(\mathbf{B}_{(\mathrm{b})}^{\dot{q}}\right)^{\mathrm{T}}$ in the mobility matrix; see Eq. (F1).

The seven coordinates $\mathbf{Q}$ require, following Eq. (12), seven random displacements $\delta \mathbf{Q}$ for every time step. But the constraint of a unit length rotation vector $\mathbf{q}$ introduces a dependence that reduces the number of independent random displacements to six. These six are conveniently sampled in the body frame following Eq. (21), with the rotation and conversion in Eq. (19) resulting in seven coupled random displacements $\delta \mathbf{Q}$. One readily shows that these $\delta \mathbf{Q}$ obey the fluctuation dissipation theorem of Eq. (15), with the mobility matrix of Eq. (F1). 
The non-cancelling terms that are retained in Eq. (19), as well as the flow-induced alignment term in the penultimate term to that equation, result in rotational displacements perpendicular to q. The quaternion vector therefore retains its length under infinitesimal time steps, but this length will gradually drift from the unit value under finite time steps. A constraint on the length is therefore included as the last term in Eq. (19), with the Lagrange multiplier solved by Eq. (18).

\section{APPENDIX G: HYDRODYNAMIC CENTER}

The values of the elements of the generalized mobility matrix will depend on the reference position $\mathbf{x}$ and the reference orientation used in the derivations of Sec. II A. For the Brownian dynamics simulations, it proves advantageous to identify the reference point with the hydrodynamic center of the cluster, i.e., the point that renders $\boldsymbol{\mu}_{\tau}^{v}$ and $\boldsymbol{\mu}_{f}^{\omega}=\left(\boldsymbol{\mu}_{\tau}^{v}\right)^{\mathrm{T}}$ symmetric. A shift of the reference point from $\mathbf{x}$ to $\mathbf{x}^{\prime}=\mathbf{x}$ $+\Delta \mathbf{x}$ can be achieved without elaborate recalculation of the grand mobility matrix: by combining Eq. (10) with Eqs. (2), (7) and (8), it can be shown that the blocks of the shifted mobility matrix (on the 1.h.s.) are related to their non-shifted counterparts (on the r.h.s.) by

$$
\begin{aligned}
\boldsymbol{\mu}_{f}^{v}\left(\mathbf{x}^{\prime}\right) & =\boldsymbol{\mu}_{f}^{v}+\boldsymbol{\mu}_{\tau}^{v} \boldsymbol{\varepsilon}_{\Delta}-\boldsymbol{\varepsilon}_{\Delta} \boldsymbol{\mu}_{f}^{\omega}-\boldsymbol{\varepsilon}_{\Delta} \boldsymbol{\mu}_{\tau}^{\omega} \varepsilon_{\Delta}, \\
\boldsymbol{\mu}_{\tau}^{v}\left(\mathbf{x}^{\prime}\right) & =\boldsymbol{\mu}_{\tau}^{v}-\boldsymbol{\varepsilon}_{\Delta} \boldsymbol{\mu}_{\tau}^{\omega} \\
\boldsymbol{\mu}_{\mathcal{E}}^{v}\left(\mathbf{x}^{\prime}\right) & =\boldsymbol{\mu}_{\mathcal{E}}^{v}-\boldsymbol{\varepsilon}_{\Delta} \boldsymbol{\mu}_{\mathcal{E}}^{\omega}+\varphi(\Delta \mathbf{x}), \\
\boldsymbol{\mu}_{f}^{\omega}\left(\mathbf{x}^{\prime}\right) & =\boldsymbol{\mu}_{f}^{\omega}+\boldsymbol{\mu}_{\tau}^{\omega} \boldsymbol{\varepsilon}_{\Delta} \\
\boldsymbol{\mu}_{f}^{\mathcal{S}}\left(\mathbf{x}^{\prime}\right) & =\boldsymbol{\mu}_{f}^{\mathcal{S}}+\boldsymbol{\mu}_{\tau}^{\mathcal{S}} \boldsymbol{\varepsilon}_{\Delta}-\boldsymbol{\psi}(\Delta \mathbf{x}),
\end{aligned}
$$

with $\varepsilon_{\Delta}=\varepsilon(\Delta \mathbf{x})$, while the remaining four blocks are unchanged. The shift to reach the hydrodynamic center is solved from

$$
\Delta \mathbf{x}=\left[\boldsymbol{\mu}_{\tau}^{\omega}-\operatorname{Tr}\left(\boldsymbol{\mu}_{\tau}^{\omega}\right) \mathbf{1}\right]^{-1}\left(\boldsymbol{\epsilon}: \boldsymbol{\mu}_{\tau}^{v}\right),
$$

with $\operatorname{Tr}$ denoting the trace. This equation also locates the hydrodynamic center of the $(6 \times 6)$ mobility matrix.${ }^{54}$ Note, however, that the $(6 \times 6)$ and $(11 \times 11)$ mobility matrices obtained via the sequence of steps in the main text in the absence and presence of stress and strain terms, respectively, in general result in two distinct hydrodynamic centers for the same body.

${ }^{1}$ G. G. Stokes, Trans. Cambridge Philos. Soc. 9, 8 (1851).

${ }^{2}$ A. Einstein, Ann. Phys. 324, 289 (1906).

${ }^{3}$ G. B. Jeffery, Proc. R. Soc. A 102, 161 (1922).

${ }^{4}$ G. K. Batchelor, An Introduction to Fluid Dynamics, Cambridge Mathematical Library (Cambridge University Press, Cambridge, UK, 2000).

${ }^{5}$ S. Kim and S. J. Karrila, Microhydrodynamics: Principles and Selected Applications, Butterworth-Heinemann Series in Chemical Engineering (Butterworth-Heinemann, Stoneham, MA, USA, 1991).

${ }^{6}$ E. Guazzelli and J. F. Morris, A Physical Introduction to Suspension Dynamics, Cambridge Texts in Applied Mathematics (Cambridge University Press, Cambridge, UK, 2012).

${ }^{7}$ H. Huang, X. Yang, M. Krafczyk, and X.-Y. Lu, J. Fluid Mech. 692, 369 (2012).
${ }^{8}$ J. Einarsson, F. Candelier, F. Lundell, J. Angilella, and B. Mehlig, Phys. Fluids 27, 063301 (2015).

${ }^{9} \mathrm{C}$. W. Oseen, Neuere Methoden und Ergebnisse in der Hydrodynamik (Akad. Verl.-Ges., Leipzig, Germany, 1927).

${ }^{10} \mathrm{~J}$. M. Burgers, "On the motion of small particles of elongated form suspended in a viscous liquid," in Second Report on Viscosity and Plasticity (Noord-Hollandsche Uitgeversmaatschappij, Amsterdam, The Netherlands, 1938), Chap. III; Reprinted in:F. T. Nieuwstadt and J. A. Steketee, Selected Papers of J. M. Burgers (Springer, Dordrecht, The Netherlands, 1995).

${ }^{11}$ J. Riseman and J. G. Kirkwood, J. Chem. Phys. 18, 512 (1950).

${ }^{12}$ V. Bloomfield, W. Dalton, and K. van Holde, Biopolymers 5, 135 (1967).

${ }^{13} \mathrm{~J}$. García de la Torre and V. Bloomfield, Biopolymers 16, 1747 (1977).

${ }^{14}$ J. García de la Torre and V. Bloomfield, Biopolymers 16, 1765 (1977).

${ }^{15}$ R. F. Goldstein, J. Chem. Phys. 83, 2390 (1985).

${ }^{16}$ J. Rotne and S. Prager, J. Chem. Phys. 50, 4831 (1969).

${ }^{17}$ H. Yamakawa, J. Chem. Phys. 53, 436 (1970).

${ }^{18}$ R. Kutteh, J. Chem. Phys. 132, 174107 (2010).

${ }^{19}$ J. Wang, E. J. Tozzi, M. D. Graham, and D. J. Klingenberg, Phys. Fluids 24, 123304 (2012).

${ }^{20}$ B. Carrasco and J. García de la Torre, J. Chem. Phys. 111, 4817 (1999).

${ }^{21}$ J. García de la Torre, G. del Rio Echenique, and A. Ortega, J. Phys. Chem. B 111, 955 (2007).

22 J. García de la Torre and B. Carrasco, Euro. Biophys. J. 27, 549 (1998).

${ }^{23}$ J. García de la Torre, D. Amorós, and A. Ortega, Euro. Biophys. J. 39, 381 (2010).

${ }^{24}$ L. Durlofsky, J. F. Brady, and G. Bossis, J. Fluid Mech. 180, 21 (1987).

${ }^{25}$ J. F. Brady and G. Bossis, Ann. Rev. Fluid Mech. 20, 111 (1988).

${ }^{26}$ W. A. Wegener, Biopolymers 23, 2243 (1984).

${ }^{27}$ J. W. Swan, J. F. Brady, R. S. Moore, and ChE 174, Phys. Fluids 23, 071901 (2011).

${ }^{28}$ Y. M. Harshe, L. Ehrl, and M. Lattuada, J. Colloid Interface Sci. 352, 87 (2010).

${ }^{29}$ M. Makino and M. Doi, J. Phys. Soc. Jpn. 73, 2739 (2004).

${ }^{30} \mathrm{~S}$. Aragon, Methods 54, 101 (2011).

${ }^{31}$ E. Dickinson, Chem. Soc. Rev. 14, 421 (1985).

${ }^{32}$ Y. M. Harshe and M. Lattuada, J. Colloid Interface Sci. 367, 83 (2012),

${ }^{33} \mathrm{H}$. Goldstein, Classical Mechanics, 2nd ed. (Addison-Wesley, Reading, MA, USA, 1980).

${ }^{34}$ N. G. van Kampen, Stochastic Processes in Physics and Chemistry (Elsevier, Amsterdam, The Netherlands, 1992), revised and enlarged edition.

${ }^{35}$ H. C. Öttinger, Stochastic Processes in Polymeric Fluids (Springer-Verlag, Berlin, Germany, 1996).

${ }^{36}$ C. Gardiner, Stochastic Methods. A Handbook for the Natural and Social Sciences, Springer Series in Synergetics, 4th ed. (Springer-Verlag, Berlin, Germany, 2009).

${ }^{37}$ S. N. Naess and A. Elgsaeter, Macromol. Theory Simul. 13, 419 (2004).

${ }^{38}$ T. R. Evensen, A. Elgsaeter, and S. N. Naess, Colloids Surf., B 56, 80 (2007).

${ }^{39}$ T. R. Evensen, S. N. Naess, and A. Elgsaeter, Macromol. Theory Simul. 17, 121 (2008).

${ }^{40}$ T. R. Evensen, S. N. Naess, and A. Elgsaeter, Macromol. Theory Simul. 17, 403 (2008).

${ }^{41}$ T. R. Evensen, S. N. Naess, and A. Elgsaeter, Macromol. Theory Simul. 18, 50 (2009).

${ }^{42}$ I. M. Ilie, W. J. Briels, and W. K. den Otter, J. Chem. Phys. 142, 114103 (2015).

${ }^{43}$ J. M. Dorrepaal, J. Fluid Mech. 84, 265 (1978).

${ }^{44}$ L. D. Favro, Phys. Rev. 119, 53 (1960).

${ }^{45}$ P.-O. Persson and G. Strang, SIAM Rev. 46, 329 (2004).

${ }^{46}$ MATLAB R2014a, The MathWorks, Inc., Natick, MA, USA, 2014

${ }^{47}$ R. W. Wilson and V. A. Bloomfield, Biopolymers 18, 1205 (1979).

${ }^{48}$ L. G. Leal and E. J. Hinch, J. Fluid Mech. 46, 685 (1971).

${ }^{49}$ S. Mueller, E. W. Llewellin, and H. M. Mader, Proc. R. Soc. A 466, 1201 (2010).

${ }^{50}$ A. Nir and A. Acrivos, J. Fluid Mech. 59, 209 (1973).

${ }^{51}$ E. J. Hinch and L. G. Leal, J. Fluid Mech. 52, 683 (1972).

${ }^{52}$ D. J. Jeffrey and Y. Onishi, J. Fluid Mech. 139, 261 (1984).

${ }^{53}$ S. Kim and R. T. Mifflin, Phys. Fluids 28, 2033 (1985).

${ }^{54}$ B. Carrasco and J. García de la Torre, Biophys. J. 76, 3044 (1999). 